Journal of

Nanotechnology and Smart Materials

\title{
Nanoplasmonics and its Applied Devices
}

\author{
Mahfuzur Rahman $\mathbf{M}^{1^{*}}$, Shahbazian-Yassar $\mathbf{R}^{2}$ \\ ${ }^{1}$ Electrical and Computer Engineering, Michigan Technological University, Houghton, MI 49931 \\ ${ }^{2}$ Mechanical Engineering-Engineering Mechanics, Michigan Technological University, Houghton, MI 49931 \\ ${ }^{*}$ Corresponding author: Mahfuzur Rahman M, Electrical and Computer Engineering, Michigan Technological \\ University, Houghton, MI 49931, USA. Email: mrahman2@mtu.edu
}

Received Date: July 11, 2014 Accepted Date: November 08, 2014 Published Date: November 26, 2014

Citation: Mahfuzur Rahman M, et al. (2014) Nanoplasmonics and its Applied Devices. J Nanotech Smart Mater 1: 1-15

\begin{abstract}
Nanoplasmonics makes a connection to conventional optics to the nanoworld. Interesting performance like subwavelength focusing to invisibility cloaking, nanoplasmonics have profound applications in science and engineering world from biophotonics to nanocircuitry. Metal and dielectric have free d-shell electrons. When metal and dielectric of different refractive index come in contact, these free electrons get accumulated in a region at the metal-semiconductor interface forming nanoplasmons. Practical implementation of nano device fabrication is the most challenging task due to the dissipative losses in metal. The optimum operating condition can be achieved by the efficient use of optical gain. We review here the ongoing progress in the field of nanoplasmonic research.

Keywords: Localized surface plasmon; Surface plasmon polaritons; Nanoparticles; Nanoplasmons; Resonance spectroscopy; Light concentrators; photovotaic device; photodectors; Metamaterial; Mach-Zender interferometric modulators; Directional-coupler switches; Hydrogel optical waveguide spectroscopy
\end{abstract}

\section{Introduction}

This paper is primarily based on the concepts of nanoplasmonics and their important application. Nanoplasmonics is a new research field for scientist for the last couple of decades. Scientists are exploring nano-structured materials for noble properties at nano scale. The interaction of light with free electrons in metal-dielectric interface causes electrons to vibrate. In optics, metals were for years believed as dull of optical properties. Once, after the discovery of surface-enhanced Raman scattering [1] metals was believed to have appreciable optical properties. Nanoplasmonics device can offer considerable exciting optical properties in near future.

When two materials of different refractive indexes come in contact, due to their difference in refractive indexes, completely free electrons in materials come across to the surface boundary of the metal - semiconductor interface. When an incident electromagnetic field exerts force on these free electrons between metal-semiconductor interfaces, these free electrons start oscillating. Depending on their nature of oscillation, surface plasmon can be of two types-Localized Surface Plasmons (LSP) and Surface Plasmon Polaritons (SPP). Typically in LSP, electrons vibrate back and forth near their position, they don't propagate. While the rest in SPP, electrons gather a considerable amount of energy and hence they propagate through the medium. These free electrons are in resonance at specific frequencies of operation; this particular frequency is defined as the resonance frequency for that device. Depending on materials used resonance behavior can be of different type though the structure, size and shape are same.

Plasmon based dielectric lenses and resonators can confined extremely high intense field in sub-wavelength. Optimum light confinement in nanoparticle can be achieved through plasmon based devices like modulators, switches, detectors, lenses, resonators.

Dissipative losses from the interaction of light with free electrons needs to be traded off with the localization with the incident light. This dissipative loss is more significant at optical frequencies like of the order of $1,000 \mathrm{~cm}^{-1}$. Researchers developed various ways to mitigate these dissipative losses. Costas M. Soukoulis et.al explained that larger the materials lesser the loss. At optical frequencies, constituent metal is responsible for major losses. Part of the losses can be eliminated by avoiding nearby resonances and sharp edges of the current flow [3, 4]. 

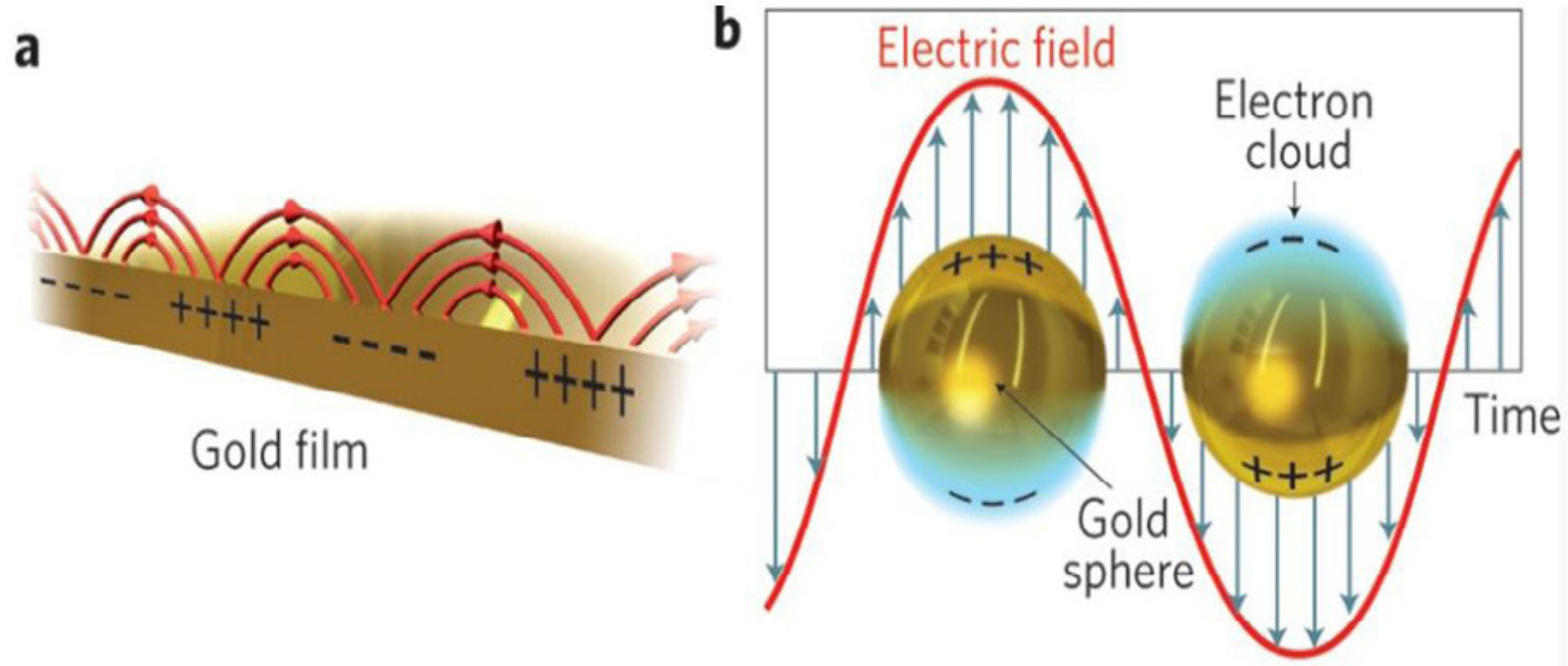

Figure 1: (a) Propagation of Surface Plasmon Polaritons (SPP), and (b) Oscillation of Localized Surface Plasmons (LSP) [2]. Reproduced with permission from Nature Photonics 5, 349-356 (2011), Nature Publishing Group.

\section{Nanomaterial and Nanotechnology}

The atom has dimension of 1 angstrom or $10^{-10}$ meter. Nano scale $\left(10^{-9}\right.$ meter $)$ materials can be considered as of several atoms and molecules. Scientist explored microstructure based materials for the decades. But nano structured material of size 1-100nm needs to be explored. Nano structured material characteristics such as lack of symmetry in electron confinement with size hinders explorations. Material properties depend on the shape and size of that material. Quantum dots are made of atoms and size of quantum dots of nano scale. Hence CdSe of different sizes have different emissions throughout the visible spectrum [5]. As shown in the figure, emission spectrum blue shifts with the decrease in quantum size. There is a direct relation between peak of the emission spectrum with the size of the quantum dot.

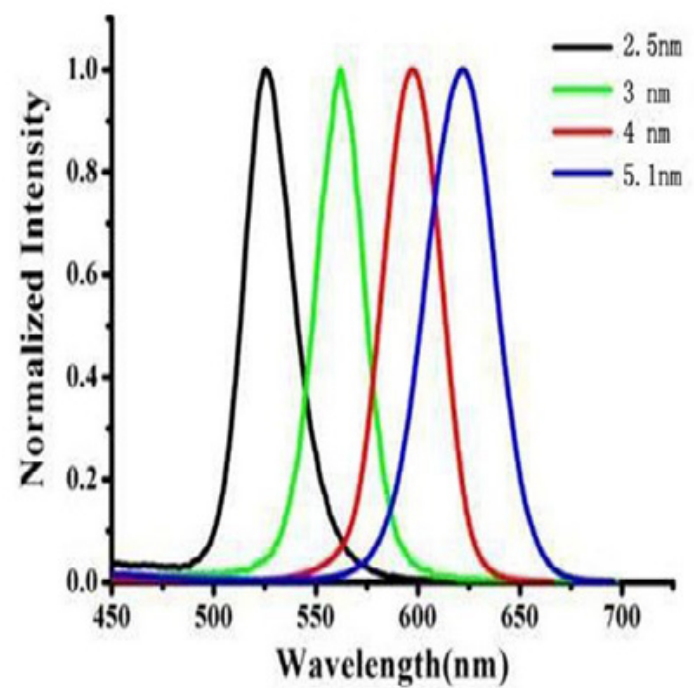

Figure 2: The fluorescence peak of CdSe with different size of quantum dots. Magnitude of intensity spectrum doesn't depend on the size of quantum dots but its operating wavelength changes with the dimension of the quantum dots. Spectrum shifts towards higher frequency (blue shift) as its dimension reduces [5]. Reprinted from book of Adv in Biomedical Eng, Vol 9 (2012), IERI. (Open Access)
Material used so far in the research of nano scale technology are copper $(\mathrm{Cu})$, silver $(\mathrm{Ag})$, gold $(\mathrm{Au})$, lead $(\mathrm{Pb})$, Indium (In), Mercury (Hg), Tin (Sn), Cadmium (Cd). Among these materials, considering optical performance and reliability, gold and silver are believed to be noble materials while copper, lead, indium, mercury, tin and cadmium are considered as secondary nanomaterials. Gold and silver nanostructures exhibit an absorption spectrum in the visible region.

As free electrons beside in the vicinity of the surface between metal-semiconductor, optical properties are controlled by the surface type-flat surface and surface with nanoparticles. The researcher has demonstrated blue shifted absorption spectrum for nano rods over the nano spheres. Not all the materials are suitable for nano devices. Materials selected for nanomaterials should have the robustness, controllable properties, unusual target binding and of course of size in nano scale. Nano structured materials has advantages over bulk material due to their target binding phenomena which can change both chemical and physical properties of nanomaterial.

To have different nanomaterials with their different shape, size and composition very well established synthesis, fabrication, and characterization methods are developed, thus allowing us excellent control over their physical and chemical properties. For specific emissive, absorptive, and light-scattering properties, sizes, shapes and compositions of nanoparticles can now be systematically varied to produce new desired nanomaterials. Scientist gained significant control both the over size [7] and surfaces [8-12] for nanoparticles. They have demonstrated that anisotropy in nanostructure like triangular prisms [13-18], nanoscale rods [19-26], nanoshells [27-31], multipods [32-34], disks [35-39] and cubes [40-42] shows better performance over solid spheres. 


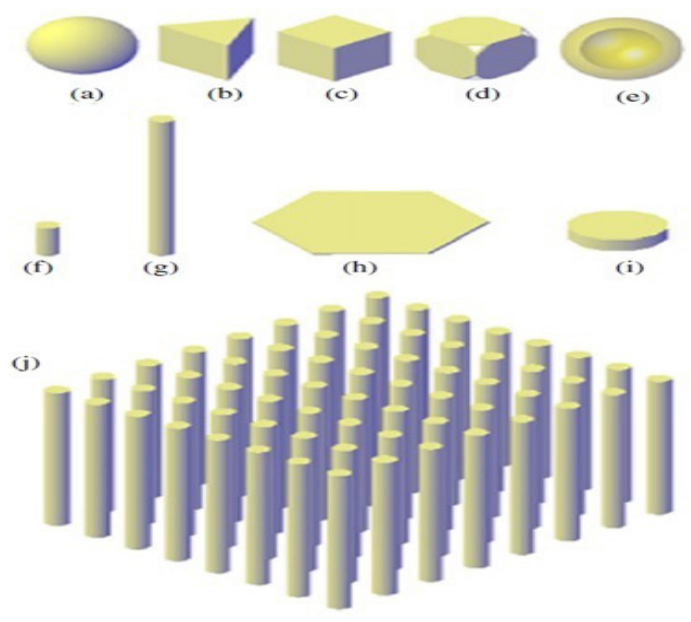

Figure 3: Schematic of metal nanostructures: (a) nanosphere, (b) nanoprism, (c) nanocube, (d) nanocage, (e) nanoshell, (f) nanorod, (g) nanowire, (h) nanosheet, (i) nanodisk, and (j) nanowire array. Reproduced with permission from Advances in Optics and Photonics, Vol. 4, Issue 2, pp. 157-321 (2012), OSA [6]

\section{Noble nanoparticles: Nanorod over nanosphere}

Color changes with the change of nanoparticle size. Gold nanosphere is characteristically red while silver characteristically yellow. This color formation is due to the oscillation of free electrons in metal-semiconductor interfaces. This free electron oscillation is in the visible spectrum and the oscillation is in strong resonance in this frequency band. At this resonance, absorption peak is at maximum as shown for gold nanoparticles [43].

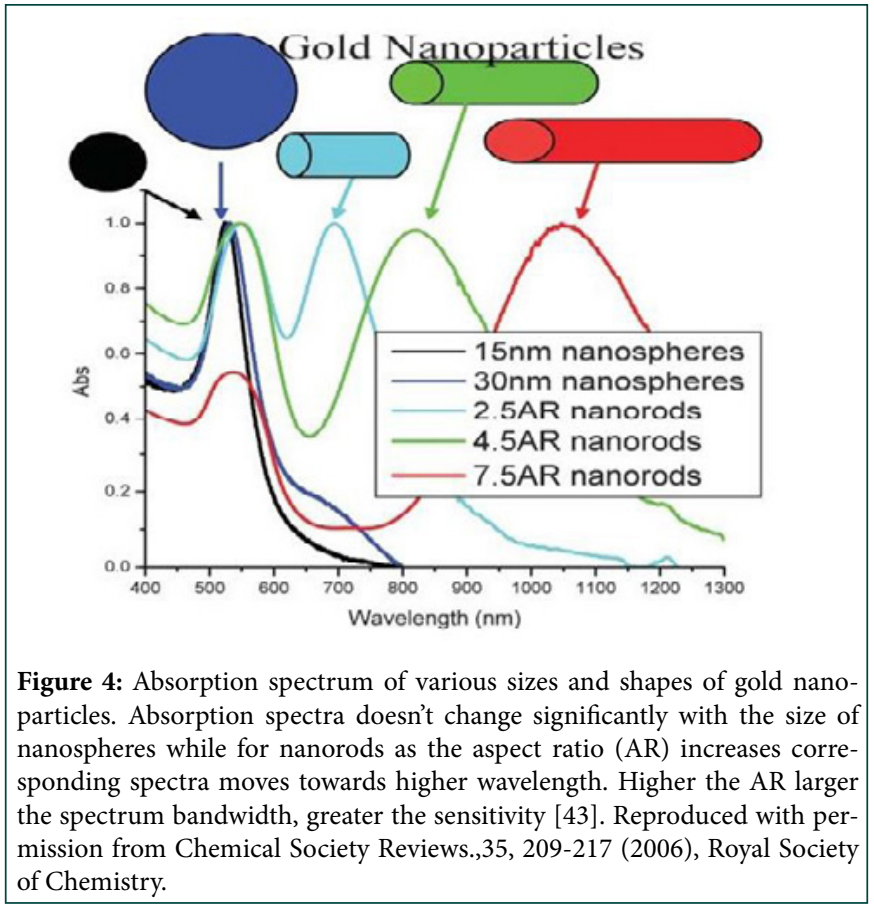

Gold nanosphere has single absorption resonance and peak of this resonance is relatively independent of the size of the gold nanospheres. With the enlargement of nanosphere its optical property changes negligibly. Where gold nanorod has two absorption resonances - one towards its shorter axis called transverse resonance second towards its larger axis called as longitudinal resonance. Optical property changes so promptly if we add anisotropy to the geometry. As we can see with the decrease in length for nanorod absorption spectra shifts towards lower wavelength making device to operate at the higher frequency causing device to a blue shift. As the orders of magnitude wider absorption peak prevail, it will promote for better sensitivity, making device for a wide range of operation.

\section{Surface Plasmon Resonance Modes}

Non-propagating vibrating electromagnetic excitations are bounded on material surfaces. And hence they are called Localized surface plasmons (LSPs). LSPs show resonance characteristic and these resonances can be of transversal and longitudinal resonance modes, dipolar or multi-polar resonance modes, Fano resonance mode. Incident electric field perpendicular to the nanostructures axis corresponds to the transversal resonance mode while electric field parallel to the axis of nanostructures matches up to the longitudinal resonance mode (Figure 5(c)). L.M. Liz-Marzan [44] investigated transversal and longitudinal resonances due to their optical anisotropy for one-dimensional nanostructures. Generally, transversal resonance mode frequency is higher than that of the longitudinal resonance mode frequency [6].

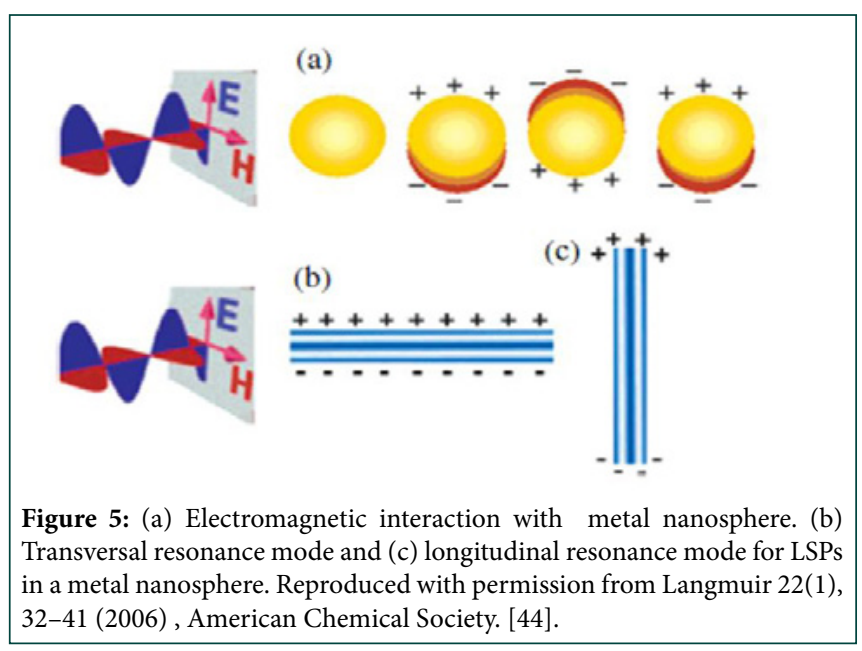

While dipolar and multi-polar resonance modes can be obtained by changing the size of one- and zero-dimensional nanostructures. Generally small size nanostructures offer dipolar resonance modes and those with large sizes exhibit multipolar resonance modes. Moreover, frequency of the multipolar resonance mode is higher than that of dipolar resonance mode. An exceptional phenomenon, Fano resonance, appears with an asymmetric line shape owing to the interactions between a superradiant "bright" mode and a subradiant "dark" mode. Interaction between dipolar and quadrupolar resonances gives rise to the Fano resonance [6].

\section{Surface Plasmon Resonance Modes}

Resonance modes can be adjusted through various nanostructure parameters like spacing, aspect ratio, and length. Wurtz et al. investigated transversal and longitudinal Localized Surface Plasmons resonance (LSPR) of Au nanostructures engineered by electrodeposition in anodic aluminium oxide (AAO) templates [45]. Figure 6(b) shows the experimental extinction spectra of Au nanostructures for various incidence angles. Incident electric field perpendicular to nanostructure axis, extinction spectra gives rise to one single 
transversal LSP peak at $520 \mathrm{~nm}$. At oblique incidence, the incident electric field which includes both s-polarized and p-polarized components exhibits two resonance peaks centered at 520 and $650 \mathrm{~nm}$ for transverse and the longitudinal resonance modes respectively. Longitudinal resonance is excited more effectively due to their strong dependence on large incidence angles. Angular sensitivity is a sign of strong anisotropy of the nanorods in the array (Figure 6(b)). Resonance peak for longitudinal resonance mode shifts towards shorter wavelengths with increasing incidence angle while angular dispersion depends on the coupling strength between nanorods [6].

Moreover, resonance mode depends strongly both on the rod aspect ratio and the distance between the nanorods in the array. An increase in the nanorod aspect ratio splits resonances into two resonance frequencies and transverse mode undergoes a blueshift, moves towards higher frequency region and longitudinal mode undergoes a redshift, moves towards low frequency region.
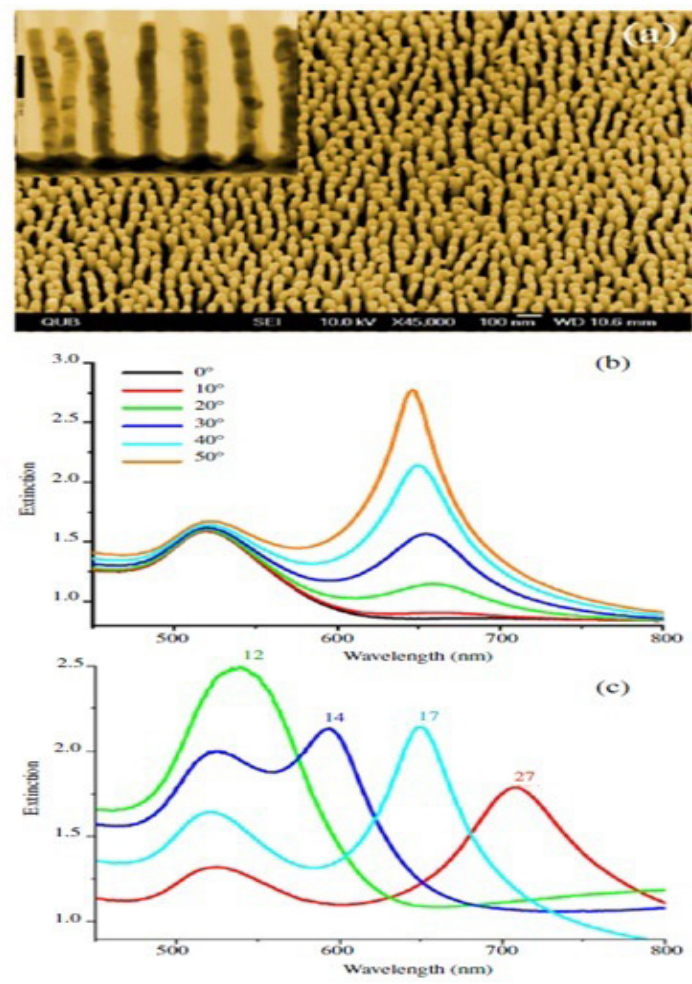

Figure 6: (a) SEM image of $\mathrm{Au}$ nanorods in air. Inset, TEM image of $\mathrm{Au}$ nanorods in anodic aluminium oxide (AAO). (b) Zero-order optical extinction spectra for different of incidence angles. The nanorod length is $300 \mathrm{~nm}$, diameter is $30 \mathrm{~nm}$, and the interrod distance is about $100 \mathrm{~nm}$. (c) Zero-order optical extinction spectra of Au nanorods in AAO as a function of rod aspect ratio. The nanorod length is $400 \mathrm{~nm}$ and the interred distance is about $100 \mathrm{~nm}$. The plots are labeled according to the nanorod's aspect ratio, corresponding to the diameters from about 15 to $30 \mathrm{~nm}$. Reproduced with permission from Opt. Express 16(10), 7460-7470 (2008), OSA [45].

\section{Dipolar and Multipolar Resonance Modes}

Dipolar and multipolar localized surface plasmon resonance modes depend on nanostructure size. For instance, spherical nanoparticles of size 5-50 nm diameter corresponds to mainly dipolar resonance, as conduction electrons in metal are in phase with the incident electromagnetic field. However, when the dimensions become long enough, multipolar resonance modes can be excited as a result of phase retardation of the applied field inside the material [46]. For example, small and larger nanorods display dipolar and multipolar resonances respectively [47-49].

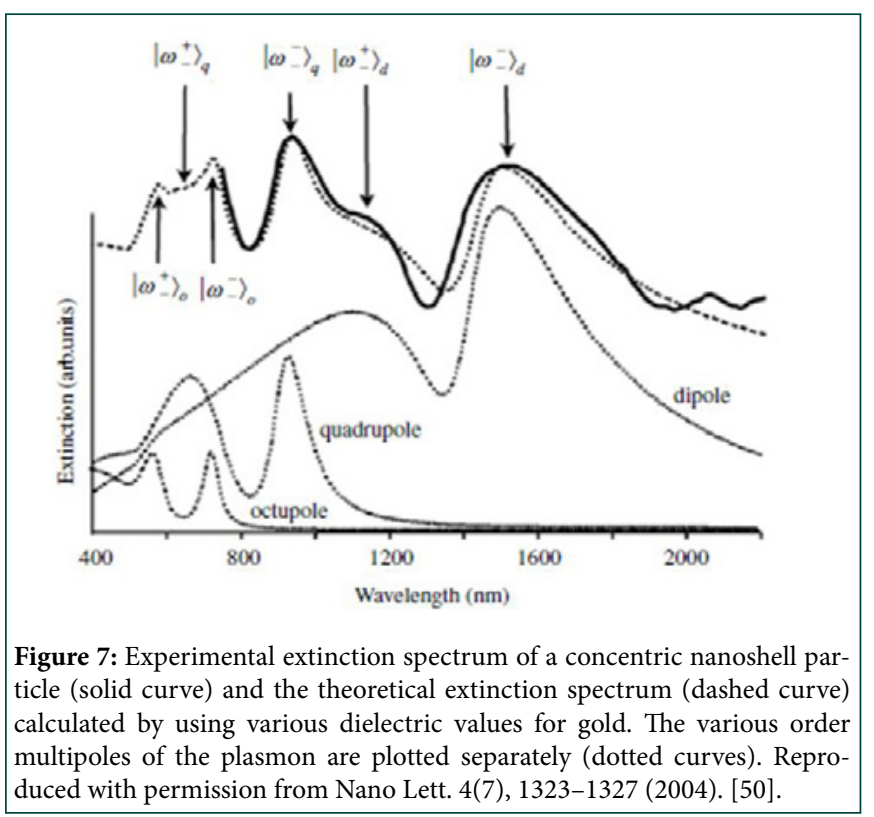

\section{Fano Resonances:}

For some systems amplitude of the oscillator increases up to its maximum when its frequency is in phase with driving force while for other systems opposite phenomenon can also occur for certain resonance condition. Let's consider weakly coupled harmonic oscillators system and an external applied force; then there will be two resonances near eigenfrequencies $\omega 1$ and $\omega 2$ of the oscillators [51]. Standard enhanced resonance exist near eigenfrequency $\omega$ - while other unusual sharp peak resonance is at eigenfrequency $\omega+$. First enhanced resonance is described by a Lorentzian symmetric profile known as a Breit-Wigner resonance, while second unusual resonance is characterized by an asymmetric profile. In 1961, Ugo Fano discovered Fano resonance exhibits a distinctly asymmetric shape resulting from the constructive and destructive interference between narrow and broad discrete resonances [52].
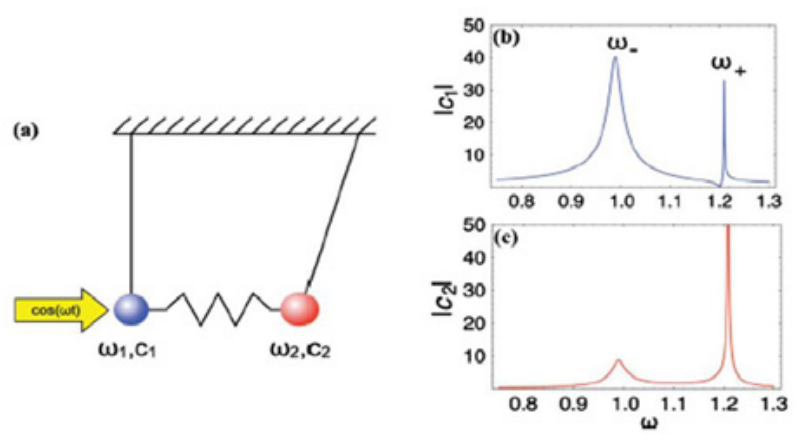

Figure 8: (a) Schematic view of two weakly coupled oscillators and a driving force (b) dependence of resonance characteristic on the amplitude of the forced oscillator $|\mathrm{c} 1|$, and (c) resonance characteristic coupled to $|\mathrm{c} 2|$. There are two resonances for these weakly coupled oscillators. The forced oscillator $(\omega 1, \mathrm{C} 1)$ exhibits resonances with symmetric and asymmetric profiles for eigenfrequencies $\omega 1=1$ and $\omega 2=1.2$ (b), respectively. The second coupled oscillator exhibits symmetric resonant profiles only (c). Reproduced with permission from Rev. Mod. Phys. 82(3), 2257-2298 (2010), APS [51] 
Due to destructive interference of oscillations between first oscillator and the external force and the second oscillator amplitude of the first oscillator reduces to zero. When the coupled oscillators system is at resonance of second oscillator there are basically two forces acting on the first oscillator, which are indeed out of phase and cancel each other. This phenomenon describes the basic properties of Fano resonance [51], namely, resonant destructive interference. (a)

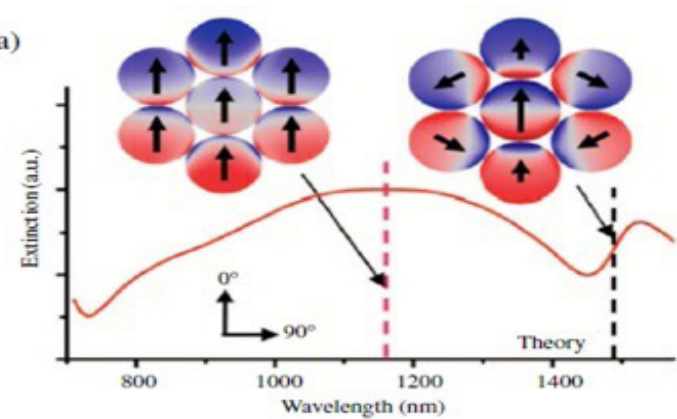

(b)

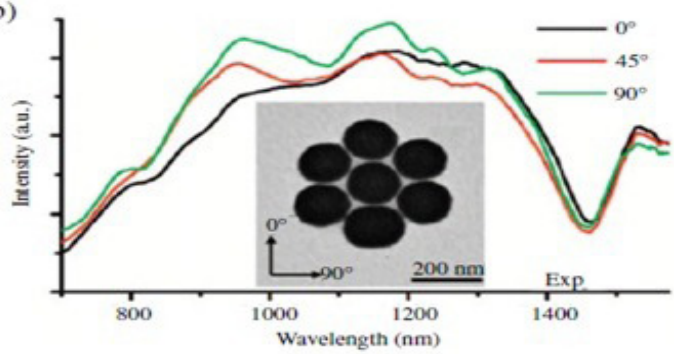

(c)

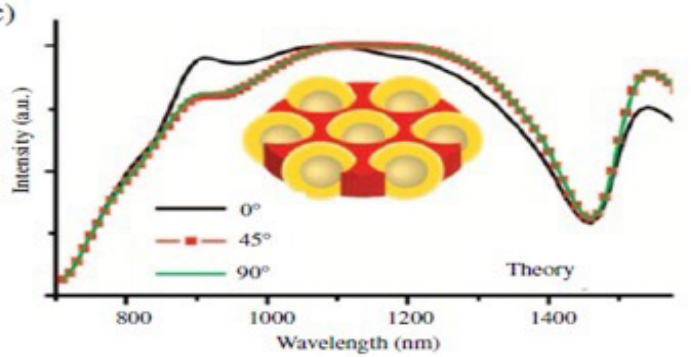

Figure 9: Fano-resonance characteristic of a plasmonic heptamer. (a) Calculated extinction spectrum and charge density plots for a heptamer excited at normal incidence. The nanoshells have dimensions $[\mathrm{r} 1, \mathrm{r} 2]=[62.5$, $85] \mathrm{nm}$, and the cluster has $1.6 \mathrm{~nm}$ gap separations and is embedded in a cylinder with a dielectric constant $\varepsilon=2.5$. The Fano minimum is at 1450 $\mathrm{nm}$. The charge density plot of the heptamer is at $1490 \mathrm{~nm}$. (b) TEM image and spectra of a heptamer at three different incident electric-field orientation angles. The nanoshells are measured to have average dimensions [r1, $\mathrm{r} 2]=[62.5,85] \mathrm{nm}$. The Fano minimum at $1450 \mathrm{~nm}$ is isotropic for these orientation angles. (c) Calculated scattering spectra for a heptamer with a geometry matching that in (a), for the three orientation angles in (b). Reproduced with permission from Science 328(5982), 1135-1138 (2010) [53].

\section{Field Enhancement through Surface Plasmons}

\section{Near-field Enhancement}

Near-field intensity is strongly enriched due to LSPP resonance near the interface between metals and dielectric materials, and the enhancement mainly depends on the shape and size of the metal nanostructures. The metal nanostructures such as nanorods, nanotips, and nanogaps show strong near-field enhancement effects. Free charge carriers are detached with the applied external electric field of the propagat- ing light. These separated charge carriers then introduce an additional field which oscillates with the same frequency as of the external field. As a result, an extremely strong field is developed near the interface of nanostructures [54]. The near-field enhancement effects has a great interest in some applications such as surface enhanced Raman spectroscopy (SERS) [5557], nonlinear optics [58-61], and nanophotonics [62-64].

\section{Transmission Enhancement}

\section{Nanohole Arrays}

Holes with sizes smaller than that of the wavelength of the incident light reveal distinctive optical properties for an opaque metal film. These holes strongly enhance the transmission of light; these fascinating effects take place due to the interaction of the light with electronic resonances in metal surfaces [65]. Output surface of the metal nanoholes act as a new point source for the light propagating through them. These transmission enhanced phenomenon through tiny holes are of great importance in the applications such as subwavelength optics, nanophotonics, optoelectronics, and sensing to biophysics [6].

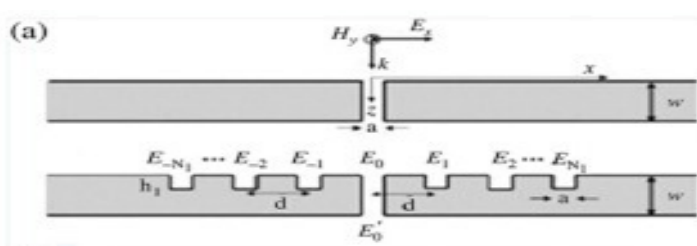

(b)

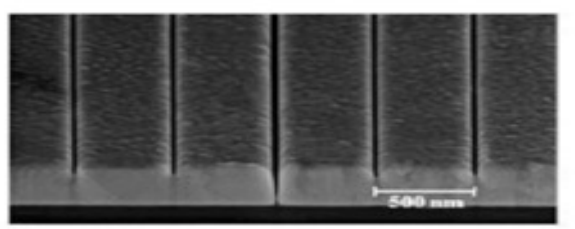

(c)

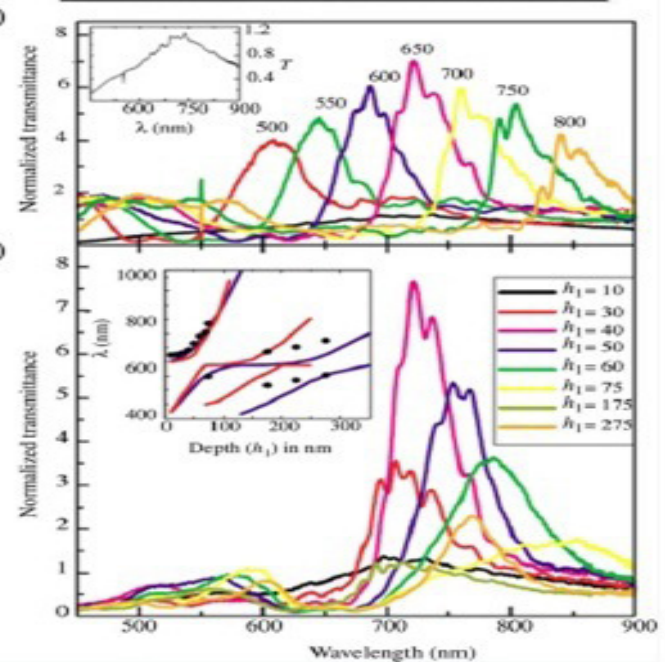

Figure 10: (a) Schematic of a single slit of width a in a metallic film of thickness $\mathrm{w}$, and single slit symmetrically surrounded in the input surface by $2 \mathrm{NI}$ grooves of depth $\mathrm{h} 1$. The separation between adjacent indentations is $\mathrm{d}$, and all groove widths are a. (b) Electron micrograph image of one of the devices analyzed and then cross sectioned by focused-ion-beam milling. Nominal values are $\mathrm{a}=404 \mathrm{~nm}, \mathrm{w}=350$ $\mathrm{nm}, \mathrm{h} 1=100 \mathrm{~nm}$, and $\mathrm{d}=500 \mathrm{~nm}$. Collection of experimental $\mathrm{T}(\lambda)$, for different structures formed by a central slit surrounded by \pm 5 grooves $(\mathrm{a}=40 \mathrm{~nm})$ on the input surface of a silver film with $\mathrm{w}=350 \mathrm{~nm}$. (c) $\mathrm{hl}=40 \mathrm{~nm}$, while the array period (d) is varied between 500 and $800 \mathrm{~nm}$. The black curve corresponds to the single-slit case (also reproduced in the inset for clarity). (d) $d=650 \mathrm{~nm}$, for different groove depths h1. In the inset, the spectral locations of measured transmission peaks (black dots) are compared with the calculated positions of the surface electromagnetic resonances of silver reflection gratings with square (blue curve) or triangular (red curve) grooves. Reproduced with permission from Phys. Rev. Lett. 90(21), 213901 (2003), APS [67]. 
While for perfect conductor these phenomenon are reversed. Considering a single hole milled in a free-standing infinitely thin Ag film. Transmission efficiency of normally incident light can be approximately expressed as [66].

$$
T=\frac{64}{27 \pi^{2}}(k r)^{4}
$$

where propagation constant, $\mathrm{k}=2 \pi / \lambda$ and $\mathrm{r}$ and $\lambda$ are the hole radius and the wavelength of the incident light, respectively. $\mathrm{T}$ is proportional to $(\mathrm{r} / \lambda)^{4}$ that indicates transmission of light is very little for a very small hole compared with the wavelength.

\section{Nanoslits}

Optical transmission like metal nanoholes, can also be enhanced through metal nanoslits. Garcia-Vidal et al. theoretically and experimentally discovered strong enhanced optical transmission through single nanoslit edged by a finite array of grooves made on a thick Ag film [67]. A single nanoslit of width of $40 \mathrm{~nm}$ was surrounded by \pm 5 grooves of length of 10 um (Figs. 10(a) and 10(b)), was fabricated by a focused-ionbeam technique. A wide transmission maxima was revealed at around $725 \mathrm{~nm}$ (Figure 10(c)). This maximum corresponds due to transmission through the nanoslit has enhancement factor of about 6. Transmission peak of grooves surrounded nanoslits of periods ranging from 500 to $800 \mathrm{~nm}$ of nominal depth of $40 \mathrm{~nm}$, shifts to higher wavelengths with enlarged period; the peak is strongest at $650 \mathrm{~nm}$. As a consequence, peak appears at the wavelength agreeing with the nanoslit waveguide mode position. For transmission enhancement optimum groove depth is of $40 \mathrm{~nm}$. Garcia-Vidal et al. suggested three main ways to enhance optical transmission: groove cavity mode excitation (depth control of the grooves), in-phase groove re-emission (period control of the groove array), and nanoslit waveguide mode (thickness control of the metal film). Two orders of magnitude transmission enhancement of light can be attained by adjusting these geometrical parameters [6].

\section{Surface Plasmon Resonance Spectroscopy}

Optical setup for Hydrogel optical waveguide spectroscopy (HOWS) [68] biosensor is depicted in Figure11. He-Ne laser with a power of $2 \mathrm{~mW}$ at a wavelength of $\lambda=633 \mathrm{~nm}$ is transmitted through a polarizer, Polarizer polarizes to transverse magnetic (TM) mode and is passed to a high refractive index $\left(\mathrm{n}_{\mathrm{p}}=1.845\right)$ prism at $[90]^{0}$ and through a sensor chip. The sensor chip consists of a glass slide and with a PNIPAAm hydrogel film and glass is coated with a gold layer of thickness between 37 and $45 \mathrm{~nm}$. Cell dimension inserts in the chip area of volume $10 \mu \mathrm{L}$, length $10 \mathrm{~mm}$ and depth $0.1 \mathrm{~mm}$. Rate of flow of liquid sample over chip is $200 \mu \mathrm{L}[\mathrm{min}]^{\wedge^{-1}}$

For the current analysis purpose, $45 \mathrm{~nm}$ gold and thiol Self-assembled monolayer (SAM) was used for the sensor. To control the angle of incidence of a laser beam $\theta$, total setup was mounted on a rotating stage. The reflected laser beam from the sensor was measured by a photo detector. Reflectivity is determined as a ratio of two light intensities: reflected light from a sensor chip and from a blank glass slide. Reflectivity variation $\sigma(\mathrm{R})$ can range from between $7 \times 10^{-5}$ and $2 \times 10^{-4}$.

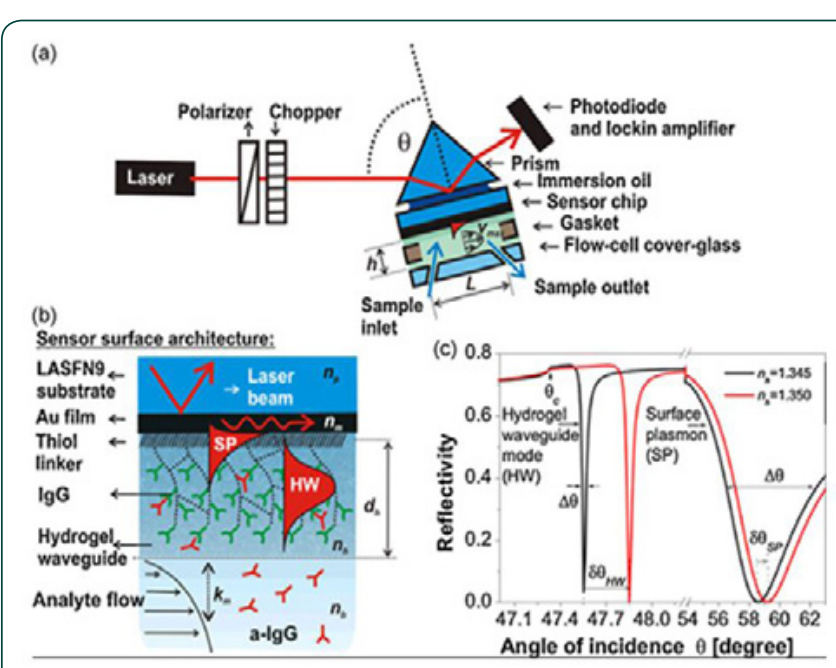

Figure 11: (a) Experimental setup for hydrogel waveguide (HW) (b) layout different surfaces and (c) reflectivity spectra for the resonant excitation HW and SP modes. Here refractive index for a hydrogel film increases $\Delta n_{h}=5 \times 10^{-3}$ from index $n_{h}=1.345$ [68]. Reproduced with permission from Biosensors and Bioelectronics 25 1663-1668 (2010), Elsevier limited.

Evanescent wave is first internally reflected at sensor surface and then penetrates through the gold layer and which is then interact with surface plasmon (SP) and hydrogel waveguide (HW) modes. This evanescent wave propagates along metal interface. SP and HW modes excite two distinct dips as can be seen in the angular reflectivity spectrum. Angles $\theta$ associated with these dips can be related with the propagation constant $\beta$ of the reflected laser beam component as

$$
[\mathrm{k}]_{0} \mathrm{n}_{\mathrm{p}} \operatorname{Sin} \theta=\operatorname{Re}\{\beta\}
$$

Where $\mathrm{k}_{\mathrm{o}}=2 \pi / \lambda$ is free space light propagation constant. Propagation constant $\beta$ of Surface Plasmon and Hydrogel Waveguide mode can be calculated from their dispersion relation.

\section{Principle of plasmon based concentrators}

Scientists are exploring nanostructures to effectively concentrate light on nanoscale devices. The structures can be of two types: resonant and nonresonant. The electric field associated with light wave in resonant structures, apply a force on the negatively charged electrons inside the metal and with this applied force, electrons oscillate, creating surface plasmon inside material. At a particular frequency this oscillation is at resonant making a huge charge displacement in contact of the metal - dielectric interface. Resonant characteristic of quasistatic and retardationbased structures will be discussed first, then will continue with nonresonant characteristic.

When the size of a nanostructure is much smaller than the freespace wavelength, i.e. $\lambda /$ a ratio is very high, then this structured nanoparticle can be called as quasistatic nanoparticle, quasistatic nanoparticle structure experiences a uniform electric field everywhere at any instant of time. With the help of potential function one can determine resonance characteristic of a given geometry. Spherical nanoparticles can be in resonance at wavelengths where $\varepsilon_{\mathrm{m}}=-2 \varepsilon_{\mathrm{d}}$, where $\varepsilon \mathrm{m}$ and $\varepsilon_{\mathrm{d}}$ are of metallic and dielectric permittivities respectively. Being 
quasistatic resonance frequencies independent of particle size, by changing metal, shape or dielectric environment resonant frequency for nanoparticles can range over a wider frequency spectrum [69] (Figure 12a, b).

Frequency depends on the energies in metal and its surrounding dielectric and this frequency will be in resonance when they are equal. Quality factor, $Q$ at the resonant frequency depends on metal losses and doesn't change with the change of geometry. Sub wavelength particles in combine can enhance field at least couple of orders of magnitude larger than single subwavelength particle. A single subwavelength particle can offer enhanced in the range of 10-100.

When nanostructures dimensions approaching external applied light wavelength. i.e. Wavelength of external light is comparable to nano particle or even smaller than nanostructure, the system is considered as retardation and effect is called as retardation effect. Retardation principle is based on scaled radio frequency antenna design concepts. Truncated SPP waveguides of wavelength scaled structures are metal nanowires [70,71] or strips [72]. Surface plasmon polaritons oscillate back and forth inside the metal, creating a standing wave in the metal. This back and forth oscillation of free electrons in metal is considered as Fabry-Perot resonator for SPPs. The resonant length of this fabry-Perot structure is equal to $\mathrm{n} \lambda_{\mathrm{sPp}} / 2$, where $\mathrm{n}$ is an integer and for first resonance mode $\mathrm{n}$ equal to one and $\lambda_{\mathrm{SPP}}$ is the wave length of the resonator (Figure $12 c-e)$.
As the structure size of this resonator is very small, dielectric lenses are used to efficiently couple freespace light to the structure of interest. Plasmonic structures can store light in areas that are sometimes quite larger than the wavelength of light.

Nonresonant effects can also be utilized to store light inside the materials. Various structured nano-devices such as plasmonic tapers: metal cones or wedges, can offer broadband, non resonant enhancements. As a wave propagates, group velocities in these structures decrease towards apex while at the same time wave vector increases towards its apex. Hence, if we launch SPP at the base of a structure, the structure will experiences strong field at its tip.

\section{Photovoltaic Devices}

For complete absorption of light photovoltaic device needs to be thick enough. Figure 13 shows AM1.5 solar absorption spectrum and light passes once through $2 \mu \mathrm{m}$ thick crystalline Si film. The figure shows that for $600-1,100 \mathrm{~nm}$ spectral range, light absorption considerably low. But traditional wafer Si solar cells have 180-300 $\mu \mathrm{m}$. For high efficiency diffusion length of minority carrier has to be several times higher than the actual material thickness.

Physical thickness of solar cell can be reduced in three ways. First, subwavelength nanoparticles interact with propagating Sun light and semiconductor thin film absorbs completely these electromagnetic waves by folding these waves

$$
\text { a }
$$

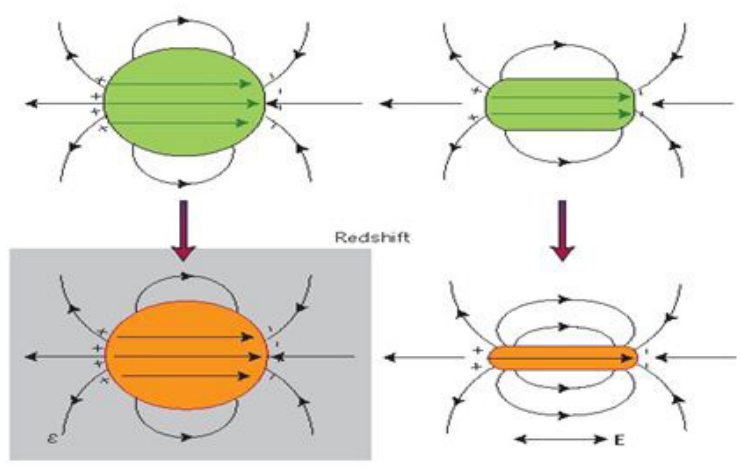

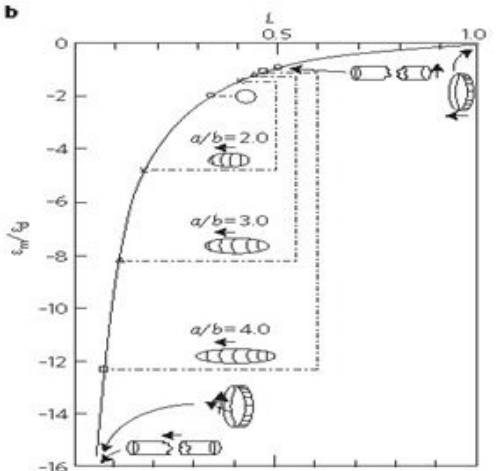
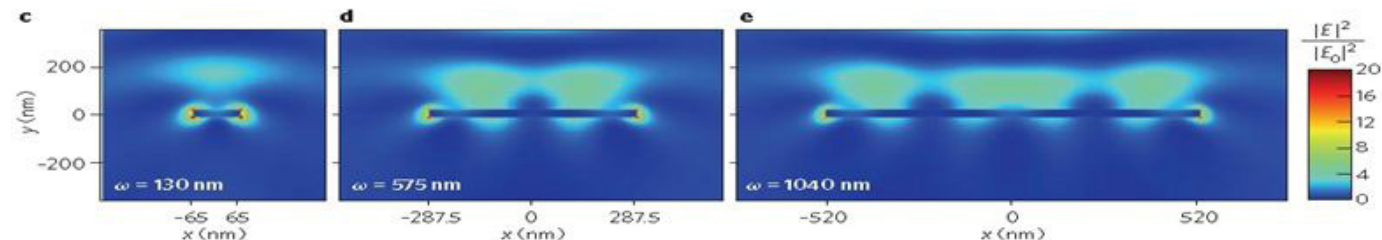

Figure 12: Resonances characteristic of different geometry and materials. a, Effects of geometry and materials on electrostatic resonances of deep-subwavelength metal nanostructures. As the surrounding dielectric constant increases the resonance for a spherical nanoparticle (shown on the left) redshifts. As the aspect ratio for a nanorod is increased the longitudinal resonance is redshifted (shown on the right). Minus (plus) signs indicate regions of high (low) electron density. b, Resonant condition $\left(\varepsilon_{\mathrm{m}} / \varepsilon_{\mathrm{d}}\right)$ as a function of aspect-ratio parameter $\mathrm{L}$ for quasistatic spheroidal particles, which are shown in the inset. The major and minor axes of the spheroid are represented by a and b, respectively. c-e, Retardation-based strip resonators. Normalized field-intensity distributions normalized to the incident intensity for the lowest odd-order resonances of 30-nm-thick silver strips that are top-illuminated with light at wavelength of $\lambda_{0}=550 \mathrm{~nm}$. E0 denotes the incident electric-field strength. Three different resonant antennas are shown that approximately measure one (c), three (d) and five (e) times $\lambda_{\mathrm{spP}} / 2$ [69]. Reproduced with permission from Nature Materials, 9, p193204 (2010), Nature Publishing Group. 


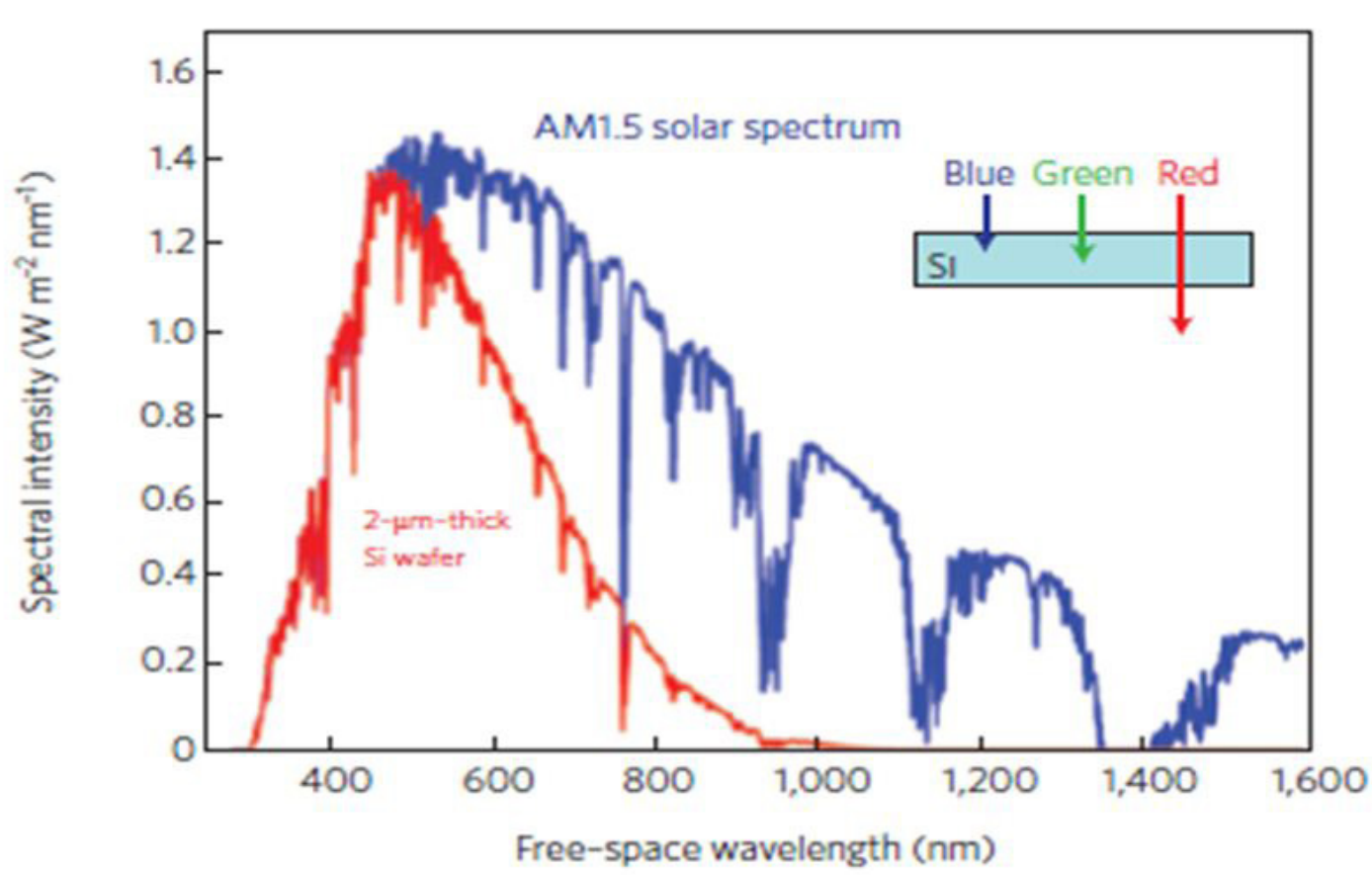

Figure 13: Absorption spectrum of solar cell. AM1.5 solar spectrum, together with a graph that indicates the solar energy absorbed in a $2-\mu \mathrm{m}$-thick crystalline Si film (assuming single-pass absorption and no reflection). Clearly, a large fraction of the incident light in the spectral range $600-1,100 \mathrm{~nm}$ is not absorbed in a thin crystalline Si solar cell. [73] Reproduced with permission from Nature mater,9, 205-213 (2010), Nature Publishing Group.

several times before being absorbed (Figure 14a). Second, subwavelength nanoparticles can be placed in metal-semiconductor interface and interacting with light, those subwavelength nanoparticles excite plasmonic near field and increases solar cell effective absorption (Figure 14b). Third, corrugated metallic film could be installed at the back of solar cell devices. Due to the refractive index mismatch between metal and semiconductor, Surface Plasmon polariton (SPP) modes generates at their interface. Absorbed sunlight could couple with these SPP modes as well as with the guided modes in the semiconductor slab (Figure 14c). Physical thickness of photovoltaic solar could be reduced considerably applying these three techniques, could be reduced in the range of 10- to 100 -fold but in both cases optical absorption remains constant.
Nanoparticles embedded inside homogeneous medium. Both forward and reverse wave propagates symmetrically from these nanoparticles. But when these nano particles beside in interface between metal and semiconductor, light penetrates first in a medium of higher permittivity. When light scattered at the critical angle, total internal reflection takes place and light remains trapped. The Si - air interface has a critical angle of $16^{\circ}$. Due to corrugated metallic surface at the back of the photovoltaic cell, the light reflected back towards the surface and again interacts with the nanoparticles and again reflects towards the corrugated back surface. Thus light bounces back and forth for several times before being absorbed in semiconductor film. Absorption efficiency depends on metal nanoparticles shape and size and it has been proved

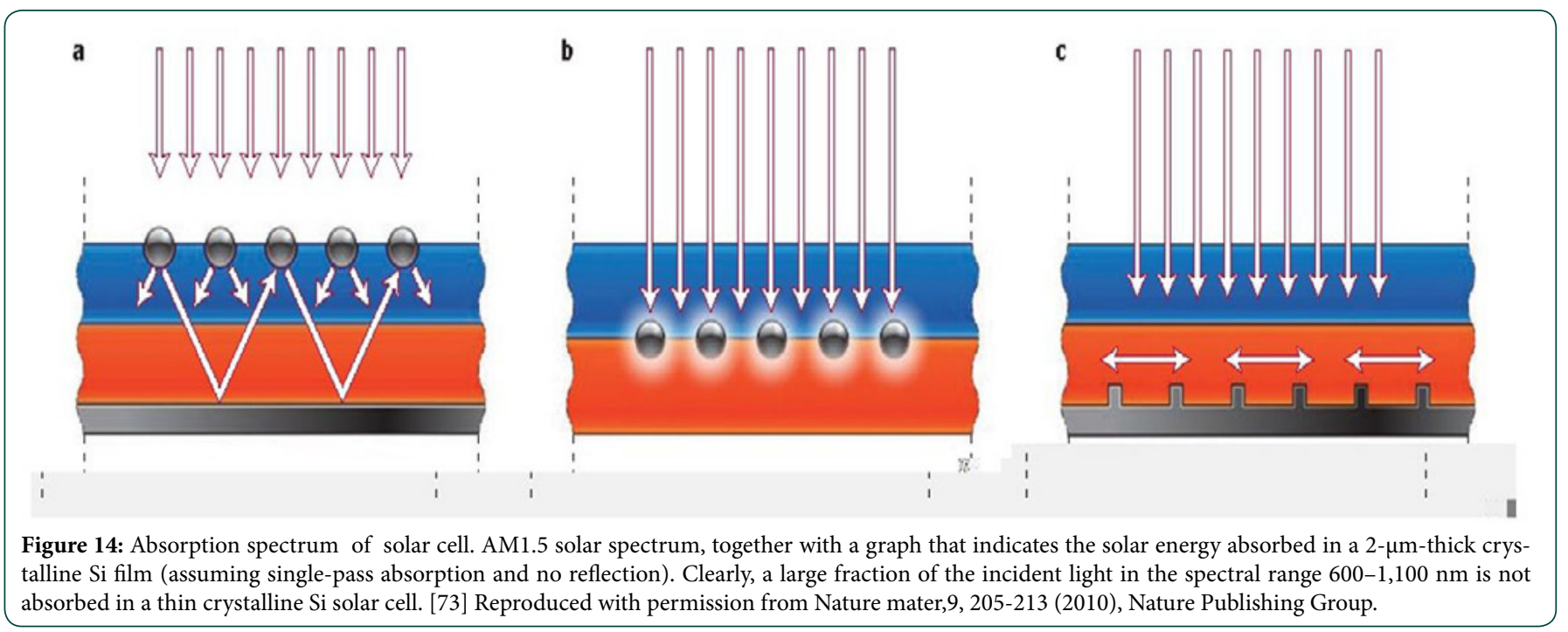


that smaller nanoparticles could increase absorb of light due to increase cross section areas [74].

\section{Optical Antenna}

Optical antenna similar to microwave and radiowave antenna, is an interesting concept to the scientists. They use optical radiation at subwavelength scale. Optical antennas can be used to enhance the efficiency of photodetection [75, 76], light emission [77, 78], sensing [79], heat transfer [80, 81] and spectroscopy [82]. Optical antenna takes care of optical propagation using elements like mirrors, lenses, fibres and diffractive elements while for radiowave and microwave antenna deals with electromagnetic fields at subwavelength scale.

Optical antenna converts optical radiation into localized energy, and vice versa. Fabrication accuracies for optical antenna necessitates down to a few nanometers. So far optical antennas have been fabricated by top-down nanofabrication techniques such as focused ion beam milling $[83,84]$ or electron-beam lithography $[85,86]$, and also by bottom-up self-assembly schemes $[87,88]$. Size of a receiver or transducer is generally much smaller than that of radiation wavelength, $\lambda$, and is normally of the order of $\lambda / 100$ and at optical frequency, antenna requires dimensions to be of $\sim 5 \mathrm{~nm}$ [89].
Strong energy concentration is observed both below the diffraction limit as well as in the semiconductor. White J.S and et al claimed these enhanced energy density below the slit due to resonance phenomena. They demonstrate this resonance characteristic as surface plasmon polariton (SPP) mode supported by the slit [see Figure16 (b)]. This resonance geometry works as a truncated metal-dielectric-metal (MDM) plasmonic waveguide [92]. A strong reflection is observed from its truncation edge terminal and cavity is termed as resonance cavity. Their proposed geometry can offer absorption enhancements up to $352 \%$ for $\lambda=633 \mathrm{~nm}$ and quite user friendly for its fabrication.

Using commercially available FDFD simulations they calculated absorption enhancement for a variable slit of dimension $1.5 \mathrm{w}$ x $50 \mathrm{~nm}$ where slit width is $1.5 \mathrm{w} \mathrm{nm}$ and height is $50 \mathrm{~nm}$. [See Figure 17 (b)]. Figure 17 (a) shows the absorption spectrum as a function of slit length as well as with slit widths, normalized to bare silicon without any metallic structure on its back; absorption enhancement decreases by $34.8 \%$ with a perfect antireflection coating with the bare silicon.

White JS et al [90] investigate scattering coefficients of the metal-dielectric-metal system (MDM) [Figure 17 (b)] for Fabry-Perot model. Plane wave electric field polarized

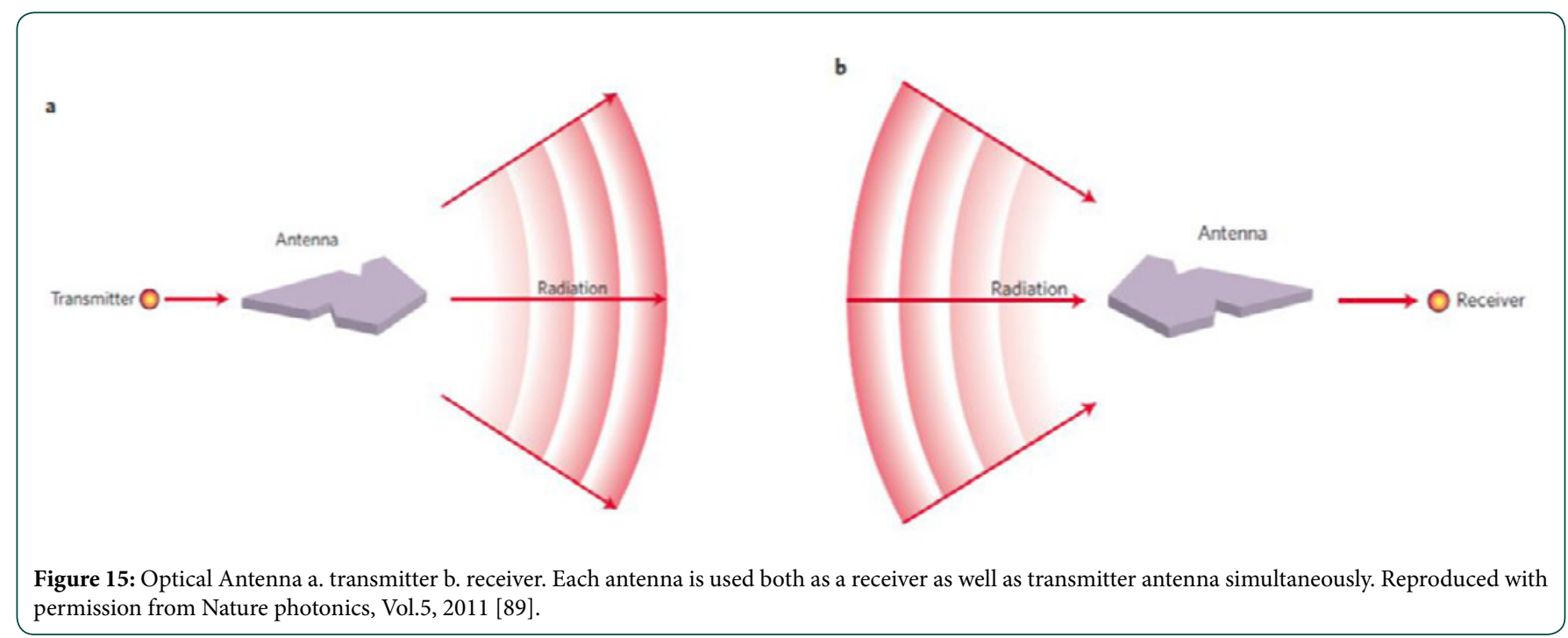

Optical antenna associates both with quantum and pure photon sources systems, and which in turn introduces new physics such as breaking of selection rules and strong coupling. Directed emission and reception concepts can now be imposed to photon emitters.

\section{Photodetectors}

White J.S and et al explored a deep subwavelength volume nanoplasmonic structure: a single isolated slit in a metallic film on an absorbing substrate [90]. They carried out their analysis based on finite-difference frequency-domain (FDFD) simulations [91] of slits generated in an Al film on a Si substrate. Figure 16 (a) shows the energy density distribution of a slit. Dimensions are for this slit $50 \mathrm{~nm}$ wide and of $100 \mathrm{~nm}$ long. The plane wave of wavelength $633 \mathrm{~nm}$ excites the structure from the top with polarization towards $\mathrm{x}$ direction. normal to $\mathrm{x}$ direction strikes the top surface with permittivity constant $\varepsilon_{1}$. Cavity $\left(\varepsilon_{2}\right)$ of length $L$ and width $\mathrm{w}$ is formed in metal film $\left(\varepsilon_{\mathrm{M}}\right)$. Plane wave couples to plasmon modes supported by the cavity with a transmission coefficient $t_{12}$. Plane wave also couples to surface plasmon polaritons on interface $\varepsilon_{1} / \varepsilon_{\mathrm{M}}$, but they have very little effect on isolated cavity and can be ignored. Incident electromagnetic waves bounce back and forth several times at top and bottom interfaces with complex reflection coefficients $r_{21}$ and $r_{23}$. Propagating plasmon mode out couples to induce absorption, which is termed as coupling coefficient $\mathrm{k}_{23}$, a ratio of absorption in the $1.5 \mathrm{wx} 50 \mathrm{~nm}$ region to the magnitude of the propagating electric field. Scattering parameters; transmission and reflection spectrum as well as coupling coefficients can be calculated from FDFD simulations. They discovered width independent first order resonance at $\mathrm{L} \approx 100 \mathrm{~nm}$ and resonance length decreases as with the decrease of slit width as $\mathrm{k}_{\mathrm{MDM}}$ increases. They also found that 
lowest order resonance length is off $\mathrm{L}_{\text {res }} \approx \lambda_{\mathrm{MDM}} / 5$. If we could eliminate losses in the aluminum film, then absorption could be increased by $19 \%(\mathrm{w}=100 \mathrm{~nm})$ to $82 \%(\mathrm{w}=30 \mathrm{~nm})$. rameters more specifically effective permittivity and effective permeability of these materials can be controlled over a wide frequency range. Metamaterial research can be motivated in the area of high-resolution imaging [94], invisibility cloaks
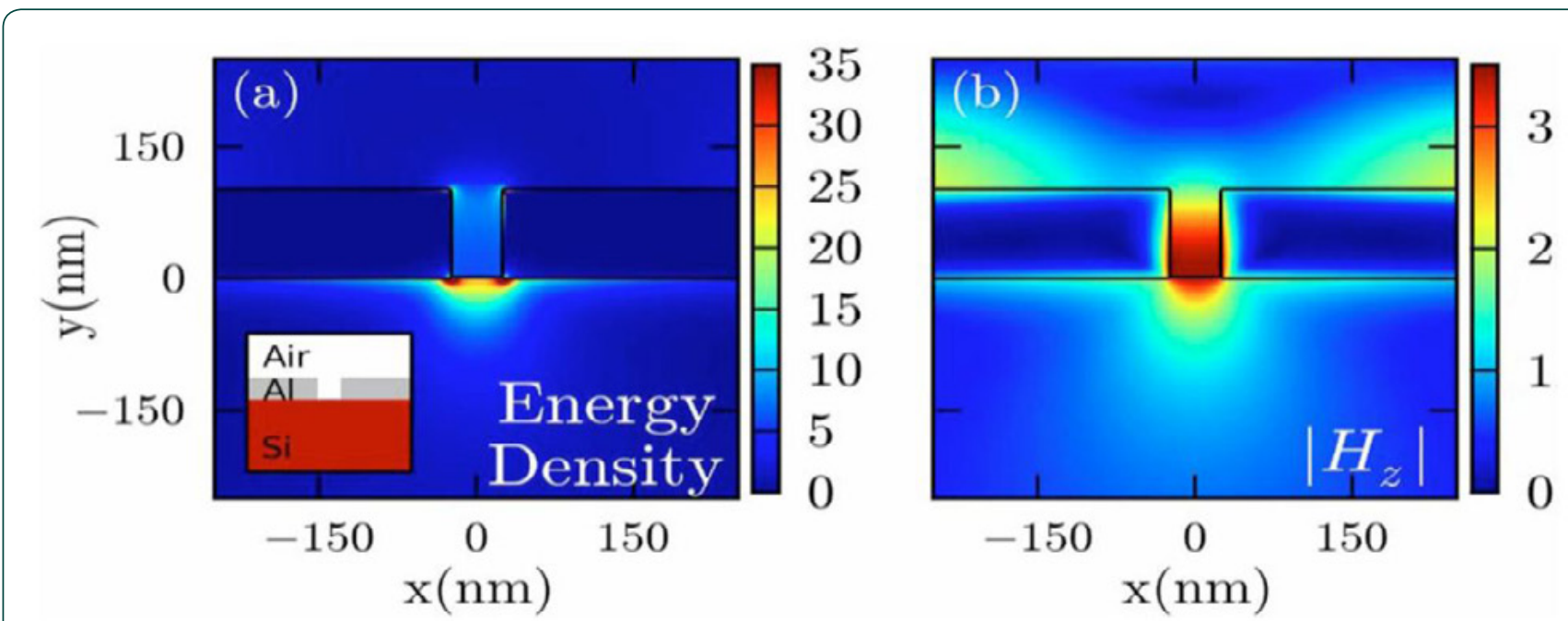

Figure 16: (a) Field energy density for a single isolated slit of $50 \mathrm{~nm}$ width in a 100-nm-thick aluminum film on a silicon substrate, the top-illuminated by a $\lambda=633 \mathrm{~nm}$ plane wave polarized in the $\mathrm{x}$ direction. (b) Magnetic field distribution for the same structure [90]. Reproduced with permission from Optics Letters 34, 686-688 (2009), Optical Society of America.

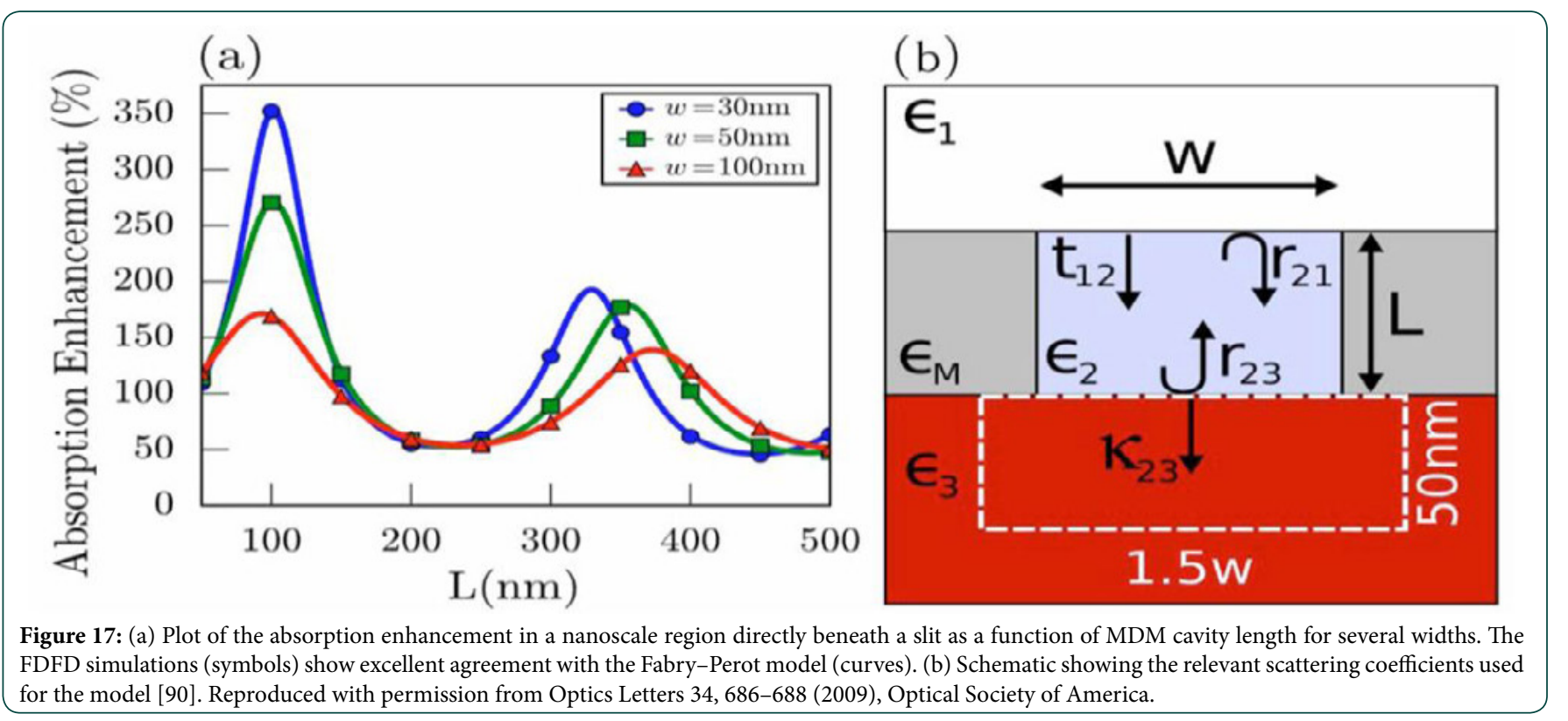

\section{Metamaterial}

Metamaterials are artificial material engineered to achieve specific electric and magnetic characteristic from that materials which are not present in nature. Exciting optical characteristic can be tuned from this man-made material. J. B. Pendry et al detailed the enhance gained plasmonic nanostructures, such as metamaterial emitters, nanolasers, spacers and so on [93]. They dealt with problems and limitation associated with these structures and resolve these problems both analytically and experimentally. They explained later the experimental success in association with the loss-compensated negative-index and double negative metamaterial. These materials are also termed as left handed materials. Effective pa-
[95], small antennas [96], quantum levitation [97].

In the last couple of decades different types of metamaterial has been introduced by numerous researchers globally. All of these metamaterials are operational in RF and optical frequency range. But these materials are lossy in the visible band and so still researchers are working to fabricate low loss metamaterial for visible and higher order spectrum. One of the key measuring factors for metamaterial characteristic is its figure of merit (FOM) and is defined as FOM = $\operatorname{Re}\{\mathrm{n}\} / \operatorname{Im}\{\mathrm{n}\}$. Higher its value, better its performance, lower its loss, easier to fabricate. 

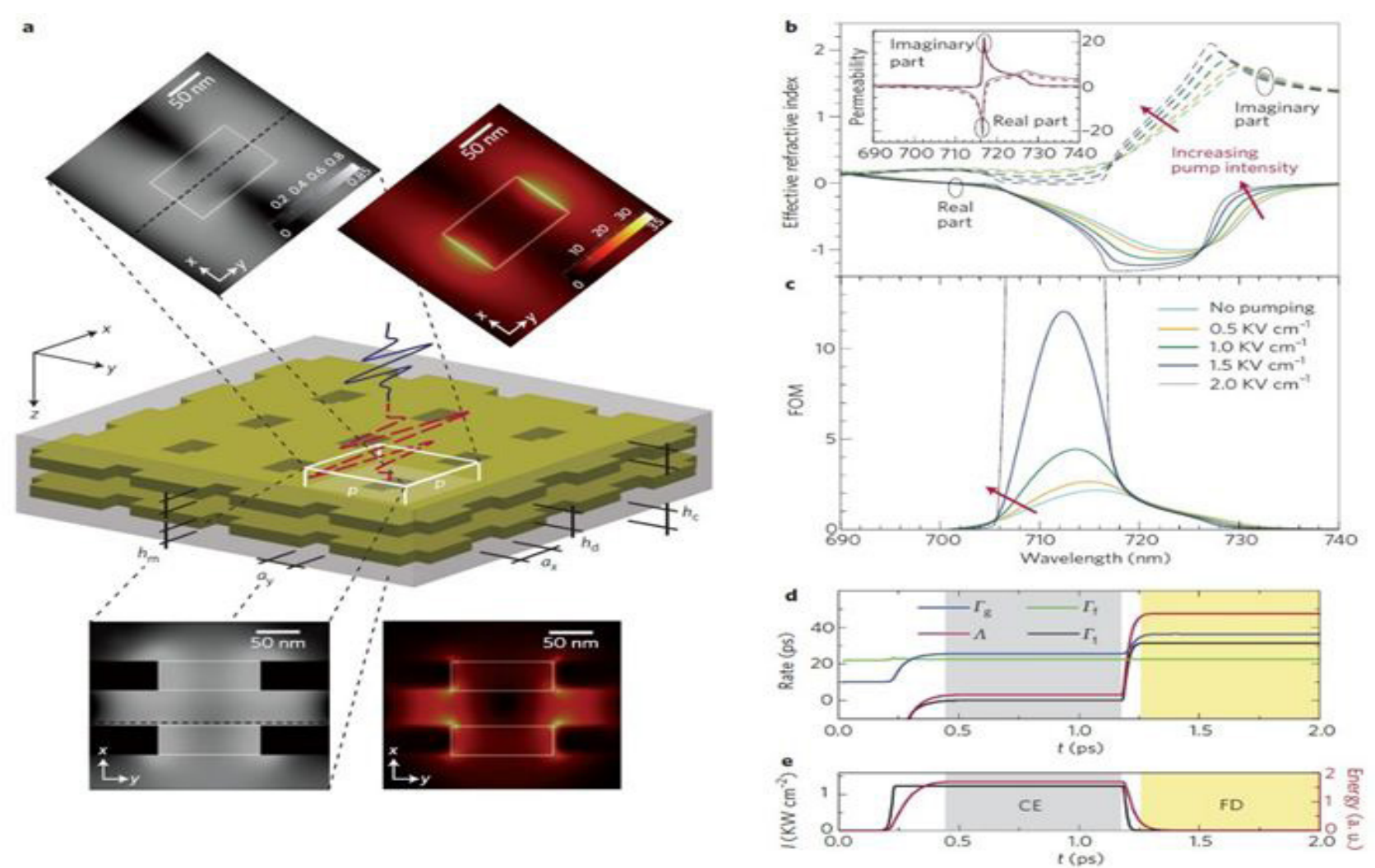

Figure 17: Negative-index metamaterials. a, Schematic of a dye-doped double-fishnet metamaterial together with exemplary profiles of the inversion (left) and electric-field amplitude (right) at the emission wavelength (710 nm) taken at the xy-plane (top) and zx-plane (bottom). Color bars: left, light (dark) shades represent high (low) areas of inversion; right, light colors represent the local field enhancement. hm, hc and hd denote the height of the metal, cladding and dielectric layers, respectively; ax and ay are the width of the rectangular holes in the $\mathrm{x}$ and $\mathrm{y}$ direction, $\mathrm{p}$ is the periodicity. The incident optical pump and probe pulses are indicated by red and blue waves. $b$, Real and imaginary part of the extracted effective refractive indices $n$ for different pump amplitudes. The peak electric-field amplitude of the pump increases in steps of $0.5 \mathrm{kV} \mathrm{cm}^{-1}$, from no pumping (cyan) to a maximum of $2.0 \mathrm{kV}$ $\mathrm{cm}^{-1}$ (black). The inset shows the real and imaginary parts of the effective permeability (black and red lines, respectively) and the corresponding results of Kramers-Kronig calculations (dotted lines) for the highest peak electric-field amplitude of $2.0 \mathrm{kV} \mathrm{cm}{ }^{-1}$. c, The figures-of-merit (FOM $=\operatorname{Re}\{\mathrm{n}\} / \mathrm{Im}\{\mathrm{n}\}$ ) for the same pumping amplitudes as those shown in b. $d$, Rate dynamics during probing in the amplifying regime of the metamaterial. Shown are the net-gain rate $\Gamma_{g}$ (blue), dissipative-loss rate $\Gamma_{f}$ (green), outflux/radiative-loss rate $\Lambda$ (red) and energy-decay rate $\Gamma_{t}$ (black). e, Dynamics of the probe-pulse intensity Is (black) and energy $U$ inside the metamaterial (red) in the regimes of continuous excitation (CE) and free decay (FD) for the active optical metamaterial of d. [93]. Reproduced with permission from Nature 11, 573-584 (2012), Nature Publishing Group.

\section{Modulators and Direction coupler Switches}

For Rapid light routing and switching in optical communication, high speed and poor efficient IC has increasing demand for the last couple of decades. In these devices light passes through a guided wave guide. The waveguide is made of core and cladding where core has a higher refractive index than that of the dielectric. Complete internal reflection takes place in core material and thus light propagates through the core material. Waveguide modes can be controlled through an external electric field for EO effects and with magnetic field for $\mathrm{MO}$ effects. Positioning of electrode for modulators or switches need to be taken special care.

Thin metal nanostripe embedded inside the dielectric can support propagation of a Long Range Surface Plasmon (LRSPP) mode, but Thomas Nikolajsen [98] and et al showed rigorously by experiment that such a stripe can also carry electrical signals that influences the LRSPP mode. They are the first guys to demonstrate the first examples of electrically controlled plasmonic components, opening new areas of research interest in photonic modulators and switches. They detailed the design, fabrication, and characterization of thermooptic Mach-Zender interferometric modulators (MZIMs) and directional-coupler switches (DCSs). These devices require low driving powers as low as $<10 \mathrm{~mW}$ for modulators and $<100 \mathrm{~mW}$ for switches, high extinction ratios as high as $>30$ $\mathrm{dB}$, moderate response times of $\sim 1 \mathrm{~ms}$.

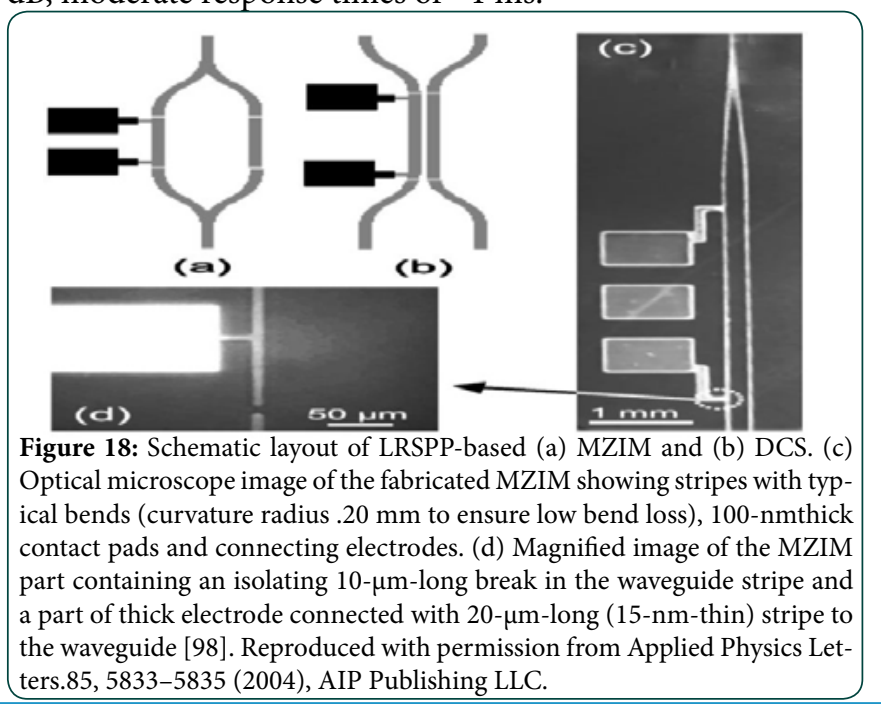


The operation of a thermo-optic MZIM is based on changing the LRSPP propagation constant in a heated arm resulting in the phase difference of two LRSPP modes that interfere in the output Y-junction. The Characteristic curve presented realizes the feature associated to LRSPPs that allow us to control and guide optical power through the same mate- the true planar processing technology, that simplifies development processes, large-scale integration possible, and photonic devices fabrication possibility.

Plasmon based MDM waveguide can be manufactured in nanoscale level. High confinement modes in the cavity is

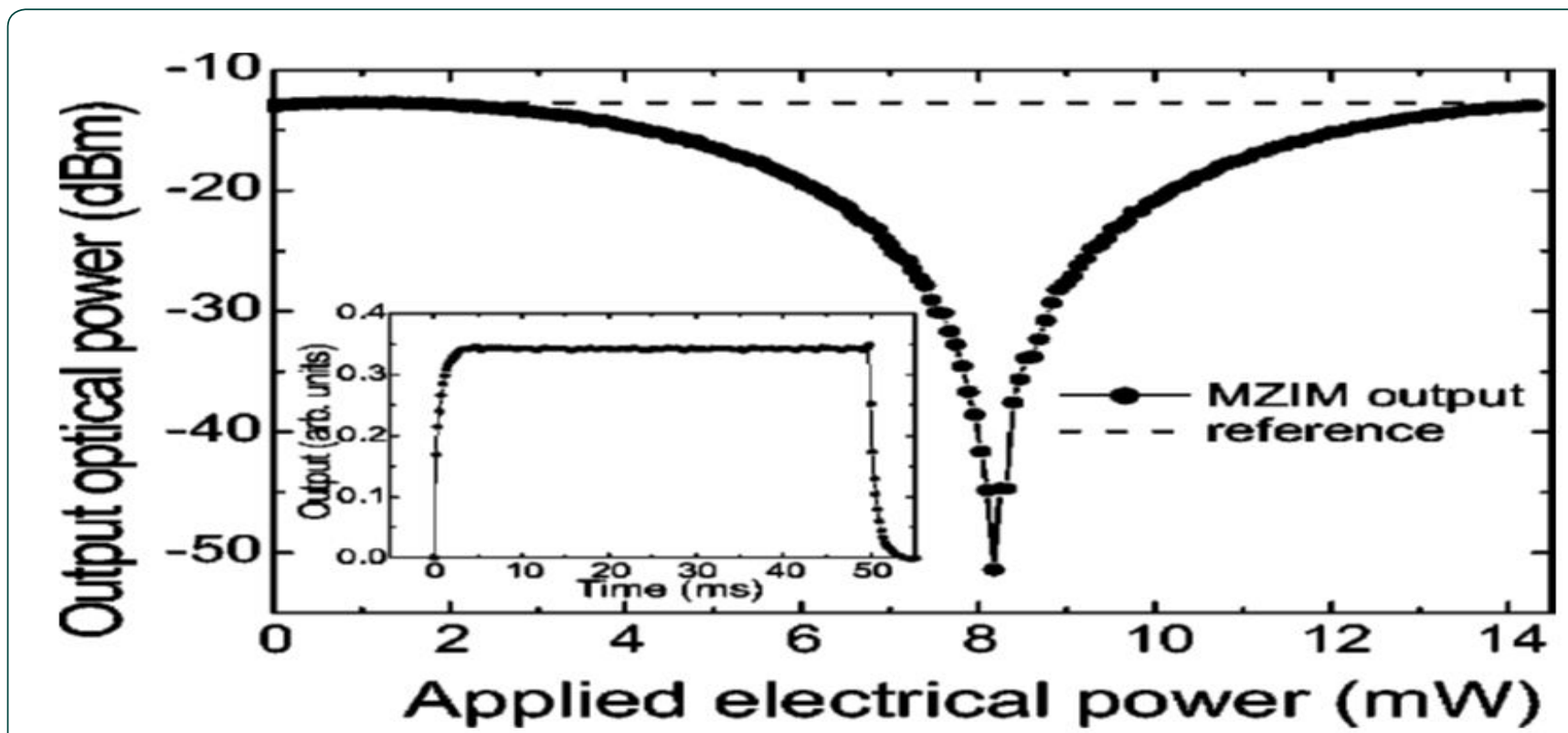

Figure 20: Dynamic characteristics of the MZIM. Inset shows the temporal response of the MZIM measured with an offset of $2 \mathrm{~V}$ and a peak-to-peak voltage of $3.8 \mathrm{~V}$ (the electrode resistance was, $1.6 \mathrm{kV}$ ). Fitting exponential dependencies to the rise and fall of the MZIM output power give a response time constant of $0.7 \mathrm{~ms}$ [98]. Reproduced with permission from Applied Physics Letters.85, 5833-5835 (2004), AIP Publishing LLC.

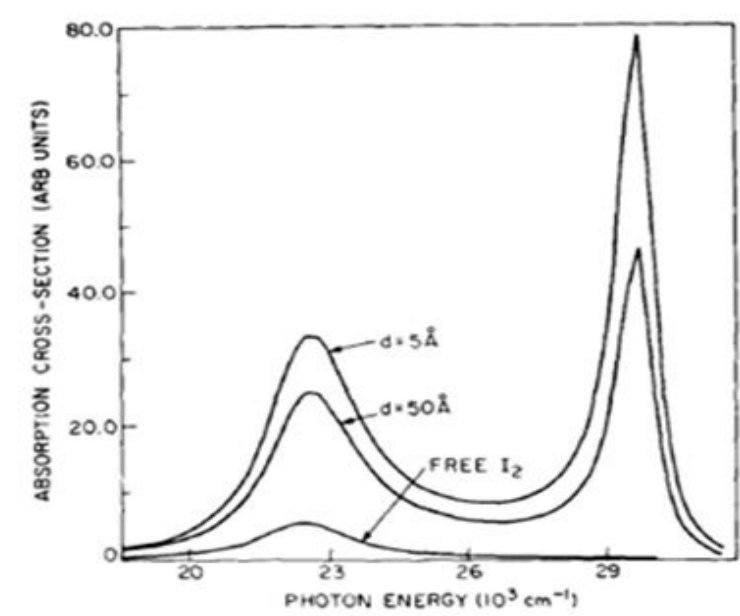

a

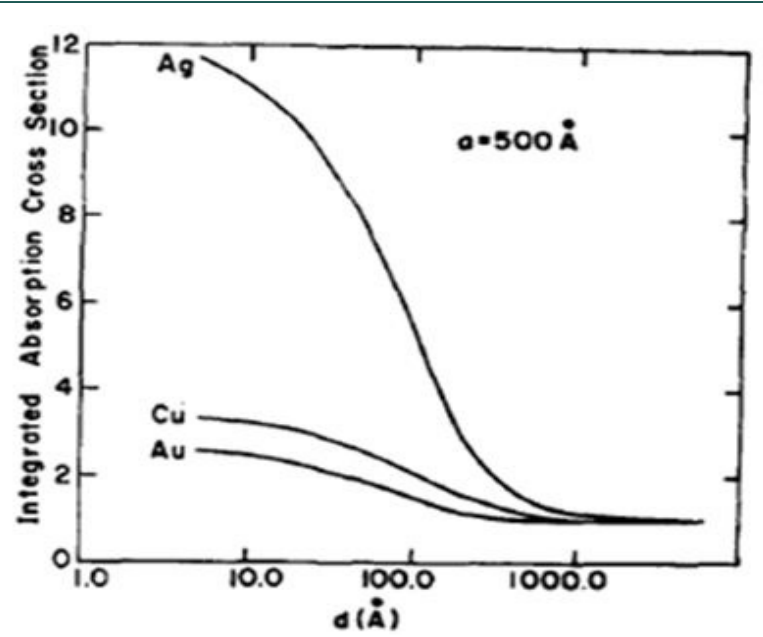

b

Figure 21: Reaction cross section is equal to the absorption cross section. a) Visible absorption cross section of a model I2 molecule near a silver sphere $\left(\mathrm{a}=500 \mathrm{~A}^{0}\right)$. The molecule is perpendicular to the surface. b)Integrated absorption cross sections for the model I2 molecule perpendicular to Ag, Cu and $\mathrm{Au}$ spheres $\left(\mathrm{a}=500 \mathrm{~A}^{0}\right)$ as a function of the molecule-surface separation. The cross sections are normalized to I when $\mathrm{d}$ very large [103]. Reproduced with permission from Journal of Chemical Physics. 75, 2205-2214 (1981), AIP Publishing LLC.

rial. Thermooptic effect depends on the type of material being used. Proper use of material will enhance system efficiency further. Characteristics presented here can be improved, the component could be made attractive for communication industries. We can use this design concept for other designs too, like Y- and X-junction based DOSs. Long range Surface plasmon polaritons (RSPP) components are fabricated through strongly limited to rapidly attenuating SPP waves. Materials used today in all optical fields have modulation amplitude of $\sim 3 \mathrm{~dB}$ and transmission losses of about $\sim 3 \mathrm{~dB}$ for IC application. Those materials are being used in all optical switching configurations [99, 100] and for all control devices [101]. In accelerating modulators researchers have demonstrated a modulator application for terahertz frequency spectrum [102]. 


\section{Nanoplasmons in Chemical and Thermal reaction}

Nanoplasmons has profound effect both on chemical and thermal reaction. Induced enhanced electromagnetic field by these nanoplasmons increases the chemical and thermal reaction rate. Abraham Nitzan and L. E. Brus [103] investigated enhanced photochemical reactions for this electromagnetic field. They demonstrated both by experimentally and numerically a simple theory for ultraviolet, visible, and infrared photochemical enhancement near rough dielectric and metallic surfaces described and investigated. Noble metals $\mathrm{Ag}, \mathrm{Au}$ and $\mathrm{Cu}$ due to their low plasma frequency are the most efficient enhancing reactors. Nitzan and Brus observed the same characteristics with alkali metals as with the noble materials. Silver is the best enhancing substrate found till to date. This is because of its narrow, pronounced plasmon resonance.

Chemical and thermal process can be controlled by the temperature induced in nanostructured particles. Heat induced in nanoplasmons has various applications like in detection and killing of cancer cells [104], drug delivery [105], photothermal melting of DNA $[106,107]$, growth of semiconductor nanowires and carbon nanotubes [108], nanofluidics and chemical separation [109], polymer surface modification [110], phase change memory $[111,112]$. Due to their large cross sectional area metallic nanoparticles are effective sources of heat generation. Absorb and scattering of light can be manipulated by changing the shape, size and dielectric environment [113]. Different methods have been developed to measure this nanoparticles temperature [114].

\section{Conclusion}

Nanoplasmonics has become one of the most exciting research areas due to the ability to manipulate free electron oscillation in the interface of metal-semiconductor in various fields and geometric configurations. These oscillations takes us to the peak of modern technology. Guiding and concentrating light capabilities in very subwavelength region of the nanostructure is the key interest of the plasmonics device.

Conventional solar cell is much more thicker than the plasmon based solar cell due to high optical length and reduced physical length. As physical length is reduced significantly much cheap plasmon solar cell can be manufactured at lower price. Modern day high resolution camera uses plasmon technology to provide us vivid pictures of the objects. In recent past in communication sectors we used very large size antenna for transmission and receiving information, with the help of nanoplasmon concepts researchers are capable of producing super small antenna. Plasmon acts as primary agent and can change the chemical and thermal reaction rate drastically. Techniques have been developed to detect infected cancer cells and then kill them in thermal plasmon treatment. We can cure infected cells with the help of drug delivery technique. In manufacturing semiconductors nanowires and carbon nanotubes nanoplasmon acts as the key role for their fabrication process. In the 60s people watched in movie actors made their appearance on screen disguise, now researchers have made invisible cloak based on these nanoplasmons that can make thing invisible. Despite of lots of remarkable properties of na- noplasmonic devices, dissipative losses in all of the conventional optical devices are considerably high. Scientist proved that engineered metamaterial can reduce this dissipative loss significantly.Larger the volume of engineered materials lower the dissipative loss.

\section{Acknowledgments}

The authors acknowledge Michigan Technlogical University for the financial support.

\section{References}

1) Fleischmann M, Hendra PJ, McQuillan AJ (1974) Raman spectra of pyridine absorbed at a silver electrode. Chem Phys Lett 26: 163-166.

2) Mathieu LJ, Maurizio R, Quidant R (2011) Plasmon nano-optical tweezers. Nature Photonics 5: 349-356.

3) Soukoulis CM, Wegener M (2010) Optical metamaterials - more bulky and less lossy. Science 330: 1633-1634.

4) Boltasseva A, Atwater HA (2011) Low-loss plasmonic metamaterials. Science 331: 290-291.

5) Yudan Hu, Li Chen, Kun Liu, Jianwen Xiong (2012) Fluorescence Properties of CdSe Quantum Dots Capped by ZnS. Advances in biomedical engineering 9.

6) Zhang J, Zhang L (2012) Nanostructures for surface plasmons. Adv Opt Photonics 4: 157-321.

7) Murray CB, Norris DJ, Bawendi MG (1993) Synthesis and characterization of nearly monodisperse $\mathrm{CdE}(\mathrm{E}=$ sulfur, selenium, tellurium) semiconductor nanocrystallites. J Am Chem Soc 115: 87068715.

8) Dabbousi BO, Rodriguez-Viejo J, Mikulec FV, Heine JR, Mattoussi H, et al. (1997) (CdSe)ZnS Core-Shell Quantum Dots: Synthesis and Characterization of a Size Series of Highly Luminescent Nanocrystallites. J Phys Chem B 101: 9463-9475.

9) Hines MA, Guyot-Sionnest P (1996) Synthesis and Characterization of Strongly Luminescing ZnS-Capped CdSeNanocrystals. J Phys Chem 100: 468-471.

10) Peng X, Schlamp MC, Kadavanich AV, Alivisatos AP (1997) Epitaxial Growth of Highly Luminescent CdSe/CdS Core/Shell Nanocrystals with Photostability and Electronic Accessibility'. J Am Chem Soc 119: 7019-7029.

11) Gao M, Kirstein S, M“ohwald H, Rogach AL, Kornowski A, et al. (1998) Strongly Photoluminescent CdTe Nanocrystals by Proper Surface Modification. J Phys Chem B 102: 8360-8363.

12) Templeton AC, Wuelfing WP, Murray RW (2000) Monolayerprotected cluster molecules. Acc Chem Res 33: 27-36.

13) Jin R, Cao Y, Mirkin CA, Kelly KL, Schatz GC, et al. (2001) Photoinduced conversion of silver nanospheres to nanoprisms. Science 294: 1901-1903.

14) Chen S, Carroll DL (2002) Synthesis and characterization of truncated triangular silver nanoplates. Nano Letters 2: 1003-1007.

15) Pastoriza-Santos I, Liz-Marz'an LM (2002) Synthesis of Silver Nanoprisms in DMF. Nano Letters 2: 903-905.

16) Sun Y, Mayers B, Xia Y (2003) Transformation of silver nanospheres into nanobelts and triangular nanoplates through a thermal process. Nano Letters 3: 675-679.

17) Yang J, Fendler JH (1995) Morphology control of pbs nanocrystallites, epitaxially grown under mixed monolayers. J Phys Chem 99: 5505-5511.

18) Jin R, Cao Y, Hao E, Metraux G, Schatz GC, et al. (2003) Controlling anisotropic nanoparticle growth through plasmon excitation. Nature 425: 487-490. 
19) Busbee BD, Obare SO, Murphy CJ (2003) An improved synthesis of high-aspect-ratio gold nanorods. Adv Mater 15: 414-416.

20) Chang SS, Shih CW, Chen CD, Lai WC, Wang CRC (1999) The shape transition of gold nanorods. Langmuir 15: 701-709.

21) Chemseddine A, Moritz T (1999) Nanostructuring titania: control over nanocrystal structure, size, shape and organization. Eur J Inorg Chem: 235-245.

22) Jana NR, Gearheart L, Murphy CJ (2001) Wet chemical synthesis of silver nanorods and nanowires of controllable aspect ratio. Chem Commun: 617-618.

23) Kim F, Song JH, Yang P (2002) Photochemical synthesis of gold nanorods. J Am Chem Soc124: 14316-14317.

24) Tang Z, Kotov NA, Giersig M (2002) Spontaneous organization of single CdTe nanoparticles into luminescent nanowires. Science 297: 237-240.

25) Xia YN, Yang PD, Sun YG, Wu YY, Mayers B, et al. (2003) Onedimensional nanostructures: synthesis, characterization and applications. Adv Mater15: 353-389.

26) Yu Y, Chang S, Lee C, Wang CRC (1997) Gold nanorods: electrochemical synthesis and optical properties. J Phys Chem B 101: 6661-6664.

27) Oldenburg SJ, Jackson JB, Westcott SL, Halas NJ (1999) Infrared extinction properties of gold nanoshells. Appl Phys Lett 75: 28972899.

28) Jackson JB, Halas NJ (2001) Silver nanoshells: variations in morphologies and optical properties. J Phys Chem B 105: 2743-2746.

29) Graf C, Blaaderen AV (2002) Metallodielectric colloidal coreshell particles for photonic applications. Langmuir 18: 524-534.

30) Oldenburg SJ, Averitt RD, Westcott SL, Halas NJ (1998) Nanoengineering of optical resonances. Chem Phys Lett 288: 243-247.

31) Jin Y, Dong S (2003) One-pot synthesis and characterization of novel silver-gold bimetallic nanostructures with hollow interiors and bearing nanospikes. J Phys Chem B 107: 12902-12905.

32) Manna L, Milliron DJ, Meisel A, Scher EC, Alivisatos AP (2003) Controlled growth of tetrapod-branched inorganic nanocrystals. Nature Mater 2: 382-385.

34) Hao E, Bailey RC, Schatz GC, Hupp JT, Li S (2004) Synthesis and optical properties of "branched" gold nanocrystals. Nano Letters 4: 327-330.

36) Hao E, Kelly KL, Hupp JT, Schatz GC (2002) Synthesis of silver nanodisks using polystyrene mesospheres as templates. J Am Chem Soc 124: 15182-15183.

37) Maillard M, Giorgio S, Pileni MP (2002) Silver nanodisk. Adv Mater 14: 1084-1086.

38) Puntes VF, Zanchet D, Erdonmez CK, Alivisatos AP (2002) Synthesis of hcp-Co nanodisks. J Am Chem Soc 124: 12874-12880.

39) Maillard M, Huang P, Brus L (2003) Silver nanodisk growth by surface plasmon enhanced photoreduction of adsorbed [ag+]'. Nano Letters 3: 1611-1615.

40) Murphy CJ (2002) Materials science. Nanocubes and nanoboxes. Science 298: 2139-2141.

41) Sun Y, Xia Y (2002) Shape-controlled synthesis of gold and silver nanoparticles. Science 298: 2176-2179.

42) Ahmadi TS, Wang ZL, Green TC, Henglein A, El-Sayed MA (1996) Shape-controlled synthesis of colloidal platinum nanoparticles. Science 272: 1924-1926.

43) Eustis S, El-Sayed MA (2006) Why gold nanoparticles are more precious than pretty gold: Noble metal surface plasmon resonance and its enhancement of the radiative and nonradiative properties of nanocrystals of different shapes. Chem Soc Rev 35: 209-217.

44) Liz-Marzán LM (2006) Tailoring surface plasmons through the morphology and assembly of metal nanoparticles. Langmuir 22: 3241.

45) Wurtz GA, Dickson W, O'Connor D, Atkinson R, Hendren W, et al. (2008) Guided plasmonic modes in nanorod assemblies: strong electromagnetic coupling regime. Opt Express 16: 7460-7470.
46) Kreibig U, Vollmer M (1995) Optical properties of metal clusters. Springer, New York.

47) Krenn JR, Schider G, Rechberger W, Lamprecht B, Leitner A, et al. (2000) Design of multipolar plasmon excitations in silver nanoparticles. Appl Phys Lett 77: 3379-3381.

48) Laurent G, Félidj N, Aubard J, Lévi G, Krenn JR, et al. (2005) Surface enhanced Raman scattering arising from multipolar plasmon excitation. J Chem Phys 122: 11102.

49) Payne EK, Shuford KL, Park S, Schatz GC, Mirkin CA (2006) Multipole plasmon resonances in gold nanorods. J Phys Chem B 110: 2150-2154.

50) Radloff C, Halas NJ (2004) Plasmonic properties of concentric nanoshells. Nano Lett 4: 1323-1327.

51) Miroshnichenko AE, Flach S, Kivshar YS (2010) Fano resonances in nanoscale structures. Rev Mod Phys 82: 2257-2298.

52) Luk'yanchuk B, Zheludev NI, Maier SA, Halas NJ, Nordlander P, et al. (2010) The Fano resonance in plasmonic nanostructures and metamaterials. Nat Mater 9: 707-715.

53) Fan JA, Wu C, Bao K, Bao J, Bardhan R, et al. (2010) Self-assembled plasmonic nanoparticle clusters. Science 328: 1135-1138.

54) Kawata S, Inouye Y, Verma P (2009) Plasmonics for near-field nano-imaging and superlensing. Nat Photonics 3: 388-394.

55) Nie S, Emory SR (1997) Probing Single Molecules and Single Nanoparticles by Surface-Enhanced Raman Scattering. Science 275: 1102-1106.

56) Kneipp K, Wang Y, Kneipp H, Perelman LT, Itzkan I, et al. (1997) Single molecule detection using surface-enhanced Raman scattering (SERS). Phys Rev Lett 78: 1667-1670.

57) Kneipp K, Moskovits M, Kneipp H (2006) Physics and Applications. Kneipp K, Moskovits M, Kneipp H (eds) Surface-enhanced Raman scattering. Springer, Berlin.

58) M"uhlschlegel P, Eisler HJ, Martin OJF, Hecht B, Pohl DW (2005) Resonant optical antennas. Science 308: 1607-1609.

59) Danckwerts M, Novotny L (2007) Optical frequency mixing at coupled gold nanoparticles. Phys Rev Lett 98: 026104.

60) Bouhelier A, Beversluis MR, Novotny L (2003) Characterization of nanoplasmonic structures by locally excited photoluminescence. Appl Phys Lett 83: 5041-5043.

61) Ghenuche P, Cherukulappurath S, Taminiau TH, van Hulst NF, Quidant R (2008) Spectroscopic mode mapping of resonant plasmon nanoantennas. Phys Rev Lett 101: 116805.

62) Oulton RF, Sorger VJ, Zentgraf T, Ma RM, Gladden C, et al. (2009) Plasmon lasers at deep subwavelength scale. Nature 461: 629-632.

63) Akimov AV, Mukherjee A, Yu CL, Chang DE, Zibrov AS, et al. (2007) Generation of single optical plasmons in metallic nanowires coupled to quantum dots. Nature 450: 402-406.

64) Noginov MA, Zhu G, Belgrave AM, Bakker R, Shalaev VM, et al. (2009) Demonstration of a spaser-based nanolaser. Nature 460: 11101112.

65) Genet C, Ebbesen TW (2007) Light in tiny holes. Nature 445: 3946.

66)Bethe HA (1944) Theory of diffraction by small holes. Phys Rev 66: 163-182.

67) García-Vidal FJ, Lezec HJ, Ebbesen TW, Martín-Moreno L (2003) Multiple paths to enhance optical transmission through a single subwavelength slit. Phys Rev Lett 90: 213901.

68) Wang Y, Huang CJ, Jonas U, Wei T, Dostalek J, et al. (2010) Biosensor based on hydrogel optical waveguide spectroscopy. Biosens Bioelectron 25: 1663-1668.

69) Schuller JA, Barnard ES, Cai W, Jun YC, White JS, et al. (2010) Plasmonics for extreme light concentration and manipulation. Nat Mater 9: 193-204.

70) Novotny L (2007) Effective wavelength scaling for optical antennas. Phys Rev Lett 98: 266802.

71) Bryant GW, García de Abajo FJ, Aizpurua J (2008) Mapping the plasmon resonances of metallic nanoantennas. Nano Lett 8: 631-636. 
72) Søndergaard T, Bozhevolnyi SI (2007) Slowplasmon resonant nanostructures: Scattering and field enhancements. Phys Rev B 75: 073402.

73) Atwater HA, Polman A (2010) Plasmonics for improved photovoltaic devices. Nat Mater 9: 205-213.

74) Catchpole KR, Polman A (2008) Design principles for particle plasmon enhanced solar cells. Appl Phys Lett 93: 191113.

75) Tang L, Kocabas SE, Latif S, Okyay AK, Ly-Gagnon D-S, et al. (2008) Nanometre-scale germanium photodetector enhanced by a near-infrared dipole antenna. Nat Photonics 2: 226-229.

76) Cao L, Park JS, Fan P, Clemens B, Brongersma ML (2010) Resonant germanium nanoantenna photodetectors. Nano Lett 10: 12291233.

77) Cubukcu E, Kort EA, Crozier KB, Capasso F (2006) Plasmonic laser antenna. Appl Phys Lett 89: 093120.

78) Pillai S, Catchpole K, Trupke T, Green M (2007) Surface plasmon enhanced silicon solar cells. J Appl Phys 101: 093105.

79) Anker JN, Hall WP, Lyandres O, Shah NC, Zhao J, et al. (2008) Biosensing with plasmonic nanosensors. Nature Mater 7: 442-453.

80) De Wilde Y, Formanek F, Carminati R, Gralak B, Lemoine PA, et al. (2006) Thermal radiation scanning tunnelling microscopy. Nature 444: 740-743.

81) Schuller JA, Taubner T, Brongersma ML (2009) Optical antenna thermal Emitters. Nat Photonics 3: 658-661.

82) Novotny L, Stranick SJ (2006) Near-field optical microscopy and spectroscopy with pointed probes. Annu Rev Phys Chem 57: 303-331. 83) Mühlschlegel P, Eisler HJ, Martin OJ, Hecht B, Pohl DW (2005) Resonant optical antennas. Science 308: 1607-1609.

84) Taminiau TH, Moerland RJ, Segerink FB, Kuipers L, van Hulst NF (2007) Lambda/4 resonance of an optical monopole antenna probed by single molecule fluorescence. Nano Lett 7: 28-33.

85) Ghenuche P, Cherukulappurath S, Taminiau TH, van Hulst NF, Quidant R (2008) Spectroscopic mode mapping of resonant plasmon nanoantennas. Phys Rev Lett 101: 116805.

86) Anika Kinkhabwala, Zongfu Yu, Shanhui Fan, Yuri Avlasevich, Klaus Müllen, et al. (2009) Large single-molecule fluorescence enhancements produced by a bowtie nanoantenna. Nat Photonics 3: 654-657.

87) Kalkbrenner T, Håkanson U, Schädle A, Burger S, Henkel C, et al. (2005) Optical microscopy via spectral modifications of a nanoantenna. Phys Rev Lett 95: 200801.

88) Anger P, Bharadwaj P, Novotny L (2006) Enhancement and quenching of single-molecule fluorescence. Phys Rev Lett 96: 113002. 89) Novotny L, van Hulst N (2011) Antennas for light. Nat photonics 5: 83-90.

90) White JS, Veronis G, Yu Z, Barnard ES, Chandran A, et al. (2009) Extraordinary optical absorption through subwavelength slits. Opt Lett 34: 686-688.

91) Veronis G, Fan S (2007) Surface plasmon nanophotonics. Brongersma ML, Kik PG (eds) Surface plasmon nanophotonics 131. Springer, Berlin.

92) Zia R, Selker MD, Catrysse PB, Brongersma ML (2004) Geometries and materials for subwavelength surface plasmon modes. J Opt Soc Am A Opt Image Sci Vis 21: 2442-2446.

93) Hess O, Pendry JB, Maier SA, Oulton RF, Hamm JM, et al. (2012) Active nanoplasmonic metamaterials. Nat Mater 11: 573-584.

94) Xu T, Zhao Y, Ma J, Wang C, Cui J, et al. (2008) Sub-diffractionlimited interference photolithography with metamaterials. Opt Express 16: 13579-13584.

95) Schurig D, Mock JJ, Justice BJ, Cummer SA, Pendry JB, et al. (2006) Metamaterial electromagnetic cloak at microwave frequencies. Science 314: 977-980.

96) Bulu I, Caglayan H, Aydin K, Ozbay E (2005) Compact size highly directive antennas based on the SRR metamaterial medium. New J Phys 7: 223.
97) Leonhardt U, Philbin TG (2007) Quantum levitation by lefthanded metamaterials. New J Phys 9: 254.

98) Nikolajsen T, Leosson K, Bozhevolnyi SI (2004) Surface plasmonpolariton modulators and switches operating at telecom wavelengths. Appl Phys Lett 85: 5833-5835.

99) Pala RA, Shimizu KT, Melosh NA, Brongersma ML (2008) A nonvolatile plasmonic switch employing photochromic molecules. Nano Lett 8: 1506-1510.

100) Dintinger J, Robel I, Kamat PV, Genet C, Ebbesen TW (2006) Terahertz all-optical molecule-plasmon modulation. Adv Mater 18: 1645-1648.

101) Dicken MJ, Sweatlock LA, Pacifici D, Lezec HJ, Bhattacharya $\mathrm{K}$, et al. (2008) Electrooptic modulation in thin film barium titanate plasmonic interferometers. Nano Lett 8: 4048-4052.

102) MacDonald KF, Sámson ZL, Stockman MI, Zheludev NI (2009) Ultrafast active plasmonics. Nat Photonics 3: 55-58.

103) Nitzan A, Brus LE (1981) Theoretical model for enhanced photochemistry on rough surfaces. J Chem Phys 75: 2205-2214.

104) Hirsch LR, Stafford RJ, Bankson JA, Sershen SR, Rivera B, et al. (2003) Nanoshell-mediated near-infrared thermal therapy of tumors under magnetic resonance guidance. Proc Natl Acad Sci U S A 100: 13549-13554.

106) Reismann M, Bretschneider JC, von Plessen G, Simon U (2008) Reversible photothermal melting of DNA in DNA-gold-nanoparticle networks. Small 4: 607-610.

107) Stehr J, Hrelescu C, Sperling RA, Raschke G, Wunderlich M, et al. (2008) Gold nanostoves for microsecond DNA melting analysis. Nano Lett 8: 619-623.

108) Cao L, Barsic DN, Guichard AR, Brongersma ML (2007) Plasmon-assisted local temperature control to pattern individual semiconductor nanowires and carbon nanotubes. Nano Lett 7: 3523-3527. 109) Boyd DA, Adleman JR, Goodwin DG, Psaltis D (2008) Chemical separations by bubble-assisted interphase mass-transfer. Anal Chem 80: 2452-2456.

110) Rontzsch L, Heinig KH, Schuller JA, Brongersma ML (2007) Thin film patterning by surface-plasmon-induced thermocapillarity. Appl Phys Lett 90: 044105.

111) Govorov AO, Zhang W, Skeini T, Richardson H, Lee J, et al. (2006) Gold nanoparticle ensembles as heaters and actuators: melting and collective plasmon resonances. Nanoscale Res Lett 1: 84-90. 112) Soares BF, Jonsson F, Zheludev NI (2007) All-optical phasechange memory in a single gallium nanoparticle. Phys Rev Lett 98: 153905.

113) Baffou G, Quidant R, Girard C (2009) Heat generation in plasmonic nanostructures: influence of morphology. Appl Phys Lett 94: 153109.

114) Baffou G, Kreuzer MP, Kulzer F, Quidant R (2009) Temperature mapping near plasmonic nanostructures using fluorescence polarization anisotropy. Opt Express 17: 3291-3298.

\section{Submit your manuscript to JScholar journals} and benefit from:

- Convenient online submission

ฯ Rigorous peer review

- Immediate publication on acceptance

- Open access: articles freely available online

9. High visibility within the field

q Better discount for your subsequent articles

Submit your manuscript at

http://www.jscholaronline.org/submit-manuscript.php 